\title{
Le risque et son évaluation subjective par le conducteur : une revue historique
}

\section{Risk and its subjective assessment by the driver: A historical review}

\author{
Jonathan Deniel ${ }^{1,3}$, Jean-Charles Bornard ${ }^{2}$, Lucie Lévêque ${ }^{1}$, Bernard Claverie $^{3}$, Thierry Bellet ${ }^{1}$
}

${ }^{1}$ Laboratoire d'Ergonomie et de Sciences Cognitives des Transports, Université Gustave Eiffel, Campus de Lyon,25, av. François Mitterrand, F-69675 Bron Cedex, France, jonathan.deniel@univ-eiffel.fr ${ }^{2}$ CIVITEC - ESI-Group, Le Récamier, 70 rue Robert, 69458 Lyon, France, jcb@esi-group.com

${ }^{3}$ ENSC - Bordeaux INP, 109 Avenue Roul, 33400 Talence, France, Bernard.claverie@ensc.fr

RÉSUMÉ. Le risque est une notion très courante : nous l'utilisons dans notre vie quotidienne et son évaluation ne demande pas d'effort de réflexion. Par exemple, lorsque nous conduisons une voiture, nous évaluons constamment le risque et utilisons cette estimation immédiate pour décider des actions à entreprendre. La plupart du temps, les humains ne sont pas pleinement conscients de ce processus d'évaluation des risques et s'interrogent rarement à ce sujet; c'est ce que nous allons explorer dans cet article. Qu'est-ce que le risque? D'où vient cette notion? Quelles sont les conceptions actuelles sur l'évaluation subjective des risques au volant? Tout d'abord, nous nous concentrerons sur la notion très générale de risque et sur son histoire. Ensuite, nous nous attarderons sur la notion d'évaluation subjective du risque (c'est-à-dire la perception du risque). Enfin, nous nous pencherons sur l'approche scientifique de l'évaluation subjective du risque par le conducteur, en passant en revue les principales théories et modèles concernant les processus cognitifs supposés à l'œuvre chez conducteur lors de l'évaluation des risques.

ABSTRACT. Risk is a very common notion : we use it in our everyday life and frequently assess it without thinking. For instance, when driving a car, one constantly evaluates risk and uses this - somewhat obvious - estimation to decide upon actions to be taken. Most of the time, humans are not even fully aware of that risk assessment process, and rarely wonder about it; this is what will be explored in this article. What is risk? Where does this notion come from ? What are current conceptions about the subjective risk assessment when behind the steering wheel? First, we will focus on the very general notion of risk and its history. Then, some time will be spent on the notion of subjective risk assessment (i.e., risk perception). Finally, we will delve into the scientific approach of driver's subjective risk assessment, with a review of the main theories and models about assumed driver's cognitive processes when evaluating risks.

MOTS-CLÉS. Situations de Conduite, Évaluation du Risque, Perception du Risque, Conscience du Risque, Modélisation Cognitive.

KEYWORDS. Driving Situations, Risk Evaluation, Risk Perception, Risk Awareness, Cognitive Modelling.

\section{Introduction}

"C'était une manœuvre risquée!", "Je ne suis pas un preneur de risques », ou même "Le risque d'accident est élevé sur cette route» sont autant de phrases qui peuvent sembler courantes. En effet, l'idée de «risque » semble naturelle aujourd'hui. Toutefois, qu'elle ne l'était pas il y a quelques siècles. Bien que le concept puisse sembler évident, nous montrerons d'abord qu'il est apparu dans un contexte très spécifique et presque anecdotique, puis qu'il s'est répandu à travers divers sujets et disciplines pour devenir ce que nous connaissons aujourd'hui. Le risque est en grande partie une question d'évaluation et d'appréciation, c'est pourquoi de nombreuses recherches ont porté sur la manière de l'évaluer. Cette évaluation peut être objective ou subjective; la première se réfère à l'évaluation des probabilités et des résultats dans le monde réel, la seconde à la façon dont les gens l'évaluent couramment (et parfois naïvement). L'évaluation générale subjective des risques étant l'objet de cet article, les principaux courants théoriques qui ont émergé pour expliquer ce phénomène seront présentés. Toutefois, l'évaluation des risques ne concerne pas seulement des questions très générales comme par exemple celles relative à industrie du nucléaire ou aux potentielles fluctuations économiques, elle concerne également ce que fait 
un conducteur lorsqu'il décide de doubler un camion, ou qu'il établit qu'il doit freiner pour éviter une collision avec un piéton. Ce type d'évaluation constante des risques intégrée dans l'activité de conduite fera l'objet des deuxième et troisième parties de cet article. De nombreux modèles ont été développés pour tenter de découvrir les processus cognitifs cachés inhérents à l'évaluation subjective du risque par le conducteur. Nous les explorerons en détail pour voir comment les chercheurs ont progressivement fusionné les processus cognitifs d'évaluation des risques avec ceux en charge de la gestion de l'activité de conduite.

\section{Généralités sur la notion de risque}

\subsection{Définition de la notion de risque}

Avant de nous intéresser à la question de l'évaluation subjective du risque et plus particulièrement à celle des processus cognitifs qui aboutissent à sa production dans le cadre de l'activité de conduite automobile, portons dans un premier temps notre attention sur la définition même de la notion de risque et sur le champ d'étude plus général de son évaluation subjective.

\subsubsection{Le risque au sens commun}

En premier lieu, rapportons la définition du terme risque telle qu'on peut la trouver à l'entrée correspondante dans le dictionnaire Trésor de la Langue Française Informatisée ${ }^{1}$.

- Le risque est subi :

- Danger éventuel, plus ou moins prévisible, inhérent à une situation ou à une activité.

- Spécialement - Éventualité d'un événement futur, incertain ou d'un terme indéterminé, ne dépendant pas exclusivement de la volonté des parties et pouvant causer la perte d'un objet ou tout autre dommage

- Le risque est affronté :

- Possibilité hasardeuse d'encourir un mal, avec l'espoir d'obtenir un bien.

Dans sa thèse Banet [BAN10] (p. 84 - 90) retrace l'étymologie de ce concept et note que, malgré le constat d'une multiplicité des définitions de la notion de risque, ce terme renvoie systématiquement à une notion de « menace pour l'homme » ou encore de « danger dont ce dernier cherchera à se prémunir ». Toutefois, dans son sens commun, le risque se distingue du danger par son caractère plus anodin, ainsi que par une plus faible probabilité d'occurrence de l'événement négatif. En résumé, le sens commun associe à la notion de risque, celle d'un danger ou d'une menace liée à une incertitude quant à sa survenue.

Cette conception, peu précise, très variable et sujette à la subjectivité, a poussé à l'établissement de définitions plus précises et scientifiques de la notion de risque 


\subsubsection{Définitions scientifiques du risque}

Les définitions plus formelles du risque sont également nombreuses et variables (en témoigne la liste établie par Macgill et Siu [MAC05]). Elle se sont multipliées au cours du temps, notamment en fonction des domaines d'application. Toutefois, on constate que la grande majorité des définitions plus techniques se réfèrent à deux notions avancées comme composantes fondamentales du concept de risque :

- La probabilité de survenue d'un événement;

— La gravité des conséquences de la survenue de cet événement;

Dans certains cas, ces deux composantes sont prises au sens mathématique, alors que dans d'autres il s'agit plus simplement de notions conceptuelles servant à la définition du terme. Il est intéressant de noter que ces deux composantes semblent apparaître dès les premières définitions scientifiques de la notion de risque.

\subsubsection{Historique de la notion de risque}

On retrace les premiers intérêts pour la notion de risque de la part des scientifiques chez Pascal et Huygens en 1657, puis chez Daniel Bernoulli et Cramer en 1738 . Dans ces deux publications, il est d'abord question de jeu de hasard [HER08]. On notera que dès cette époque, les mathématiciens font le constat du "manque de rationalité" des individus dans leur appréhension du risque, des pertes et des gains . Le paradoxe de Saint-Pétersbourg présenté par Cramer et N. Bernoulli et dont traite l'écrit de D. Bernoulli en témoigne [BER38].

Ce paradoxe repose sur le principe d'un jeu dont les possibilités de gains sont infinies et pour lequel la réponse mathématique préconise au joueur de miser toutes ses économies disponibles. Or, il s'avère que spontanément aucun joueur ne verse vers cette option. Ce constat paradoxal a servi de source au développement de l'étude scientifique des choix et des risques qui aboutira plus tard à la théorie des jeux dans le domaine économique. Il constitue probablement le premier constat objectif de l'existence d'une forme de biais des sujets concernant l'évaluation des risques et des gains (dans ce cas particulier il s'agit de ce qui sera établi plus tard sous le terme d'aversion pour le risque [HER08]. Ces écrits anciens produisent déjà une définition mathématique formelle incluant les probabilités d'un événement et ses conséquences.

La formule de calcul du risque est alors définie comme suit :

$$
r=\frac{\Sigma p_{i} \times C_{i}}{1}=\frac{\Sigma p_{i} \times C_{i}}{\Sigma p_{i}}
$$

où $p_{i}$ renvoie à la probabilité d'un événement $i$ et $C_{i}$ à la conséquence (positive ou négative) de sa survenue.

Cette formule indique que le risque est défini comme le barycentre des événements pondérés par leur probabilité d'occurrence. La mathématisation, dans un but d'objectivation et de quantification du risque, a donné naissance à une discipline spécifique : la cindynique. 
La notion de risque n'est pas restée cantonnée au simple domaine des jeux, elle a ensuite été évoquée dans le domaine de la navigation avant de s'étendre plus largement au cadre économique et assurantiel [HER08].

\subsection{Bilan de la notion générale de risque}

L'exploration des définitions du risque et de leur historique, nous informe que deux aspects majeurs présentent une relative constance :

- Les composants de la notion de risque :

- La probabilité de l'événement

— Les conséquences de l'événement

- L'évaluation du risque :

- Aspect objectif du risque

- Aspect subjectif du risque

Malgré une grande variété apparente, des constantes anciennes persistent donc dans l'élaboration de la définition du risque. Elles renvoient à des dimensions pouvant relever d'évaluations objectives comme subjectives. Comme nous l'avons vu, initialement, les besoins les plus importants concernaient l'économie et les assurances, et ont nécessité le développement de méthodes d'évaluation objective du risque aboutissant à l'émergence de la cindynique et par la même occasion des experts de cette discipline. Toutefois, nous avons pu constater que dès les premières approches de la notion de risque, son évaluation subjective par des profanes a montré quelques particularités l'éloignant des évaluations produites par les experts.

Concentrons-nous à présent sur ce domaine particulier qu'est celui de l'étude de l'évaluation subjective du risque. Dans notre contexte, notre intérêt principal concerne la dimension subjective de l'évaluation du risque. Dans ce domaine, de nombreuses recherches et de nombreuses découvertes ont été faites. La part la plus importante de ces travaux concerne l'évaluation subjective du risque au sens général. La prochaine section évoque les principaux travaux et auteurs de ce domaine.

\subsection{L'évaluation subjective du risque ou Perception du Risque}

La perception du risque est un terme consacré, qui renvoie à l'évaluation subjective du risque par les individus dans divers contextes. Nous reprenons ici ce terme pour traiter des travaux généraux consacrés à l'évaluation subjective du risque, puis nous l'abandonnerons au profit d'une terminologie selon nous plus adaptée lorsque nous traiterons des travaux liés au domaine de l'étude du conducteur et de son évaluation du risque.

\subsubsection{Aperçu historique de la notion de Perception du Risque}

Initialement, la différence entre l'évaluation subjective et objective du risque est apparue comme une curiosité. Les premières recherches sur ces questions ont été la source du développement d'une approche mathématique dans laquelle une estimation des valeurs (gains ou pertes) et une estimation des probabi- 
lités (des événements considérés) est effectuée pour servir de base au calcul du risque. Cette approche a ensuite été développée et axiomatisée par Von Neuman et Morgenstern en 1947, puis étendue sous le nom de paradigme de l'utilité subjective espérée. Dans ce paradigme, le sujet est considéré comme un acteur rationnel.

Plus tardivement, le développement de certaines technologies complexes (i.e. énergie nucléaire, industrie chimique, etc.) a rendu plus manifeste la différence entre l'évaluation effectuée par les experts (i.e. cindynique) et celle produite par les individus de la population au travers de l'opinion publique. La question de l'évaluation subjective du risque est alors devenue une problématique à part entière.

Starr fut le premier à proposer une méthode d'étude de la perception du risque : la méthode des préférences révélées [STA69]. Ces premiers travaux se situent dans le cadre conceptuel considérant l'individu comme un agent rationnel, et concluent que les différences observées entre les experts et les profanes proviennent potentiellement d'un manque d'information de ces derniers. Les recherches dans le domaine ne s'arrêteront pas là. D'autres chercheurs apporteront des critiques à ces conclusions et proposeront de nouvelles approches.

\subsubsection{La théorie des perspectives}

Au cours des années qui suivirent, Kahneman et Tversky [KAH11], prennent la suite de ces premiers travaux et mettent à jour un certain nombre de particularités de l'évaluation subjective du risque, en l'étudiant dans le contexte de la théorie économique dominante de l'époque, basée sur l'utilité espérée. Leur but était de vérifier si les agents économiques prennent des décisions rationnelles face au risque (i.e. suivant l'utilité espérée). Leurs travaux ont été couronnés de succès et ont mis à jour différents effets aujourd'hui fort connus, tels que ceux de supériorité des pertes, d'aversion ou d'attirance pour le risque ou encore de renversement des préférences [RAU01]. Basée sur ces travaux, la théorie des perspectives servira, entre autres, de socle au développement de l'économie comportementale.

\subsubsection{Système 1, Système 2, heuristiques et biais}

L'approche se basant sur la théorie de l'utilité espérée, suppose un examen de toutes les options et de tous les cas de figure. Or, il apparaît que dans les situations naturelles, comme par exemple celle d'un diagnostic médical, même les spécialistes ne se concentrent sur l'examen détaillé que d'un nombre restreint d'options, souvent identifiées extrêmement rapidement. Kahneman, Epstein et nombre d'autres chercheurs [SLO04] proposent alors un modèle cognitif impliquant deux voies de traitement de l'information. L'une rapide, intuitive, peu coûteuse en ressources cognitives et souvent implicite (le système 1), l'autre plus lente, rationnelle, coûteuse en ressources cognitives et souvent explicite (le système 2) [KAH11]. La voie rapide mettrait en place un mode de raisonnement particulier appelé heuristique et caractérisé par cette grande vitesse d'exécution et ce faible coût cognitif. C'est ainsi que l'hypothèse d'une influence lourde des raisonnements de type heuristiques dans l'évaluation des risques a été avancée comme une approche explicative des tendances constatées chez les sujets dans l'évaluation des risques. Ces heuristiques seraient la source des déformations constatées dans l'évaluation (i.e. aversion au risque ou préférence pour le risque, supériorité des pertes, etc.) et qualifiées de biais cognitifs.

D'autres chercheurs se sont investis dans ce champ d'étude et ont produit d'importants travaux, comme 
par exemple le développement du paradigme psychométrique initié par Solvic [SLO87], que nous n'évoquerons que superficiellement ici. Par ailleurs Cadet et Kouabénan [CAD05] dans une revue de la littérature sur le sujet, rapportent le développement d'une autre approche qu'ils nomment paradigme cognitif.

\subsubsection{Le paradigme psychométrique}

Lorsque le constat d'une divergence régulière entre l'évaluation produite au moyen des méthodes objectives et les évaluations subjectives (en particulier produite par les profanes) a été fait, les méthodologies employées se basaient principalement sur une démarche consistant à produire des évaluations de risque puis à en détecter les divergences, erreurs et tendances particulières en comparaison de l'évaluation guidée par la démarche purement rationnelle. Pourtant, détecter et décrire ces tendances, désaccords et leurs directions n'était pas suffisant; il fallait également les expliquer.

L'une des méthodologies développées pour cela a consisté à mesurer la manière dont les sujets classaient les risques les uns par rapport aux autres c'est la méthode dite des préférences révélées et exprimées [STA69], [SLO87]. En complément de cette première procédure Slovic et ses collaborateurs ont aussi décidé de s'intéresser aux évaluations quantitatives des sujets concernant un grand nombre d'aspects et de caractéristiques supposées associées aux risques. Cette nouvelle méthodologie, appelée paradigme psychométrique, ne se contentait plus simplement de chercher à décrire les "erreurs" produites dans les évaluations du risque ou des probabilités mais s'intéressait à la composition sous-jacente de cette évaluation. L'idée étant de découvrir s'il existait des dimensions ou des caractéristiques ayant une association particulière à l'évaluation du risque [HER08], [SLO87].

Le paradigme psychométrique fait le choix du recours aux analyses factorielles pour identifier des éléments communs sous-jacents aux diverses évaluations subjectives du risque par les profanes. Slovic [SLO87] identifie ainsi trois facteurs importants structurant l'espace d'organisation des risques subjectivement évalués chez les sujets non experts :

- Les risques effrayants

- Les risques inconnus

- Le nombre de personnes exposées (facteur plus secondaire)

La prise en compte de ces dimensions générales permet de prédire les évaluations de risques qui seront effectuées par les sujets suivant le type d'événement considéré. Ainsi, plus un événement négatif sera effrayant, inconnu et concernera de nombreuses personnes plus il sera subjectivement évalué comme risqué par les sujets.

Slovic et ses collaborateurs ont appliqué la même méthodologie aux évaluations de risques fournies par les experts. L'analyse produit un résultat très différent en révélant que le niveau de risque estimé par les experts est simplement corrélé au facteur de la mortalité annuelle attendue pour les événements évalués. Ces nouveaux résultats éclairent donc une partie de la différence entre l'estimation du risque par le grand public et celle des experts. La différence d'estimation entre le grand public et les spécialistes concernant, par exemple, l'énergie nucléaire devient ainsi bien plus compréhensible. À ces trois dimensions détectées vient ensuite s'ajouter ultérieurement une quatrième : le potentiel de signal qui désigne la capacité d'un accident à avoir des répercussions qui se diffusent dans la société. Le potentiel de signal est propre à chaque risque (accident potentiel) et n'est pas nécessairement lié au nombre de victimes. Ainsi par 
exemple, un incident nucléaire ne faisant aucune victime (e.g. Three Miles Island) pourra avoir de très grandes répercussions dans la société qu'un autre bien plus pourvoyeur de mortalité comme par exemple les accidents de la route ou encore les épidémies de grippe saisonnières. Hermand et Chauvin [HER08] dans leur revue de la littérature sur les apports du paradigme psychométrique font état d'un grand nombre de recherches effectuées entre 1978 et 2005 venant confirmer ces découvertes.

Le développement du paradigme psychométrique a donc permis de découvrir de nouveaux résultats éclairant les particularités de l'évaluation du risque par les sujets non experts. Slovic [SLO87], [SLO04] conclue, au travers de la phrase "On trouve à la fois de la sagesse et des erreurs dans les attitudes et perceptions du public", que si l'évaluation des risques par les experts est précise et informée, l'évaluation des profanes est riche malgré un plus faible niveau conceptuel, les deux évaluations ne semblent en fait pas structurées de la même manière.

\subsubsection{Le paradigme cognitif}

Historiquement, le paradigme cognitif s'est développé en parallèle du paradigme psychométrique. Selon Cadet et Kouabénan [CAD05], les précédents paradigmes et approches développées se consacrent à l'étude d'évaluations produites de risque, de ce qu'ils appellent un résultat stabilisé; en d'autres termes, du produit final des processus d'évaluation du risque. Les notions de système 1 , système 2 , d'heuristiques et de biais ne sont pas les seules conceptions qui ont été proposées.

Dans leur revue, ces auteurs présentent le modèle de Hogarth [HOG80] - ici rapporté dans la figure suivante - comme l'un des plus pertinents. Ils indiquent qu'une des premières propriétés intéressantes du modèle consiste en la différenciation entre la sortie produite (i.e. évaluation du risque) et l'utilisation qui en sera faite au travers de l'action puis de son résultat. Ils indiquent également que ce modèle présente l'avantage de considérer l'évaluation comme produite dans un contexte, dans un environnement qui pourra potentiellement jouer un rôle. Ils insistent enfin sur le recours au concept de schéma, qui permet de résoudre un certain nombre de problématiques soulevées, par une une approche plus séquentielle et orientée du traitement de l'information. En effet, une conception de la chaîne de production de l'évaluation du risque sous la forme simple d'une collecte importante d'informations, suivie de la production d'une évaluation, pose de nombreuses difficultés, à commencer par la potentielle surcharge du système. Les schémas - décrits par Amalberti [AMA96] comme des structures de données destinées à représenter des situations génériques - permettent, en tant que cadres orientés vers un but, de résoudre cette problématique, en définissant les informations pertinentes à rechercher et en limitant les possibilités de choix d'action au travers de règles à appliquer.

Le recours à la notion de schéma implique donc que, dans un premier temps, le sujet identifie le cadre (i.e. schéma) qui guidera le processus d'évaluation. Ce schéma prescrira des sources d'informations requises limitant ainsi la collecte et privilégiant la qualité des sources d'information à leur quantité. Le schéma bornera ensuite les processus d'évaluation et de décisions d'action suivant le but vers lequel il est orienté. Cadet et Kouabénan relèvent également les limitations consécutives à l'utilisation de schémas dans le cadre de la perception du risque, en particulier le cas du choix d'un schéma inapproprié dont la conséquence est d'orienter l'évaluation dans une mauvaise direction et qui peut se traduire par une difficulté du sujet à prendre conscience de son erreur et à la corriger en activant le schéma pertinent.

Suivant cette approche, l'évaluation des risques ne serait donc pas un processus simple et séquentiel 


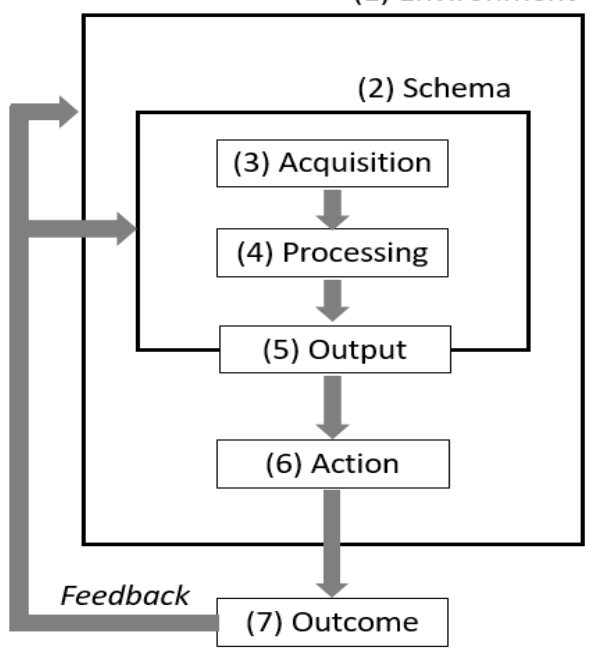

Figure 1. : Modèle conceptuel du jugement de Hogarth (1980) (issu de [CAD05])

basé sur une collecte aussi exhaustive que possible d'informations, suivie de la production d'une évaluation, mais un processus plus complexe et cyclique, basé à la fois sur des activités de collecte limitées d'informations, d'inférences et de vérifications d'hypothèses. Cette conception n'est pas sans conséquences, elle implique par exemple qu'il faille un moyen de limiter le nombre d'informations à prendre en compte, ce qui peut se faire au travers de l'établissement d'une sélection des sources supposées porteuses d'informations de plus grande valeur. Cette nécessité de hiérarchiser les sources d'informations suivant leur utilité serait l'une des caractéristiques expliquant les différences entre l'évaluateur profane de l'évaluateur expert. Ce dernier ayant, par la formation et la pratique, un avantage dès les premières étapes du traitement.

Cadet et Kouabénan abordent également une autre difficulté à surmonter dans l'analyse et la compréhension des processus de l'évaluation du risque : les modalités d'intégration des informations. En effet, les indices collectés peuvent être de natures variables, le système cognitif doit pourtant les agencer pour produire une valeur unique et homogène. Cette procédure est nommée intégration.

Cette approche par modélisation cognitive de l'évaluation du risque n'est pas incompatible avec les autres approches développées dans ce champ de recherche. On constate d'ailleurs que les conceptions de Kahneman et celles de Slovic convergent d'une certaine façon et que tous les auteurs aboutissent à la proposition de mécanismes cognitifs pour rendre compte de l'évaluation subjective du risque.

La théorie des heuristiques s'est également fortement développée, elle a été entre autres, alimentée par les résultats issus de la méthode psychométrique. Parmi ces résultats, l'identification du rôle joué par les émotions et les affects dans les processus d'évaluation du risque a pris de l'importance dans les conceptions plus récentes, comme en témoigne l'heuristique d'affect proposée par Slovic.

\subsubsection{L'heuristique d'affect}

Les travaux effectués dans le cadre du paradigme psychométrique ont été poursuivis et approfondis. Il en a résulté la découverte, que le sentiment de peur représente la dimension prépondérante de l'évaluation subjective du risque; ce qui a amené Slovic à faire le lien entre ses résultats et les propositions évoquées 
plus haut concernant les deux voies de traitement de l'information et de production de jugements de risque avancées par Kahneman et Tversky. Il en vient ainsi à qualifier la voie rapide (i.e. système 1), qu'il nomme système expérientiel, comme marquée par les émotions et les affects, le sentiment de peur prenant toute sa place au sein de cette conception dans le contexte de l'évaluation subjective du risque. Il propose alors la notion d'heuristique d'affect dans laquelle il rattache ses travaux à ceux de Damasio et à sa théorie des marqueurs somatiques [DAM94], [SLO04]. Il montrera notamment que la force de l'affect (positif ou négatif) impacte l'évaluation des bénéfices et des risques.

Finucane, sa collaboratrice, montrera en outre, que la relation inverse entre les bénéfices et les risques perçus est grandement impactée par la contrainte temporelle. En d'autres termes, moins le sujet dispose de temps, plus la relation inverse entre les bénéfices évalués et les risques perçus sera forte, ce qui, une nouvelle fois, est un argument en faveur du recours au système expérientiel et de l'importance de l'affect lors les évaluations subjectives du risque [SLO4].

Nous disposons maintenant d'une vision plus claire des conceptions développées dans le cadre de l'évaluation du risque de manière générale. Nous avons vu que certaines recherches se concentrent sur des évaluations très spécifiques et artificielles (e.g. jeux); d'autres s'intéressent aux évaluations du risque concernant une grande variété d'objets ou d'événements (e.g. industrie du nucléaire, de la chimie, tabagisme, etc.); enfin certaines s'intéressent aux évaluations du risque en conditions naturelles sur des objets précis et parfois complexes, comme le pilotage d'un avion ou encore un diagnostic médical. L'évaluation du risque dans le domaine de la conduite automobile n'a pas fait exception à la recherche sur ce sujet. Dans les sections suivantes, nous porterons notre attention sur les recherches spécifiquement dédiées à cette thématique en passant en revue les différents modèles cognitifs et les principaux résultats qui ont été produits.

\section{Les modèles cognitifs de l'évaluation du risque par le conducteur}

En conduite automobile, comme dans d'autres domaines où peuvent s'exercer le jugement et l'évaluation du risque, différents niveaux peuvent être identifiés. En effet, comme indiqué précédemment, l'évaluation subjective du risque peut s'exercer sur un spectre allant des aspects très généraux de cette activité, comme par exemple l'estimation du risque routier en général, aux aspects très particuliers comme l'évaluation du risque à un instant, un endroit et dans un contexte particulier. Un certain nombre de modèles ont vu le jour au cours des dernières décennies; il nous faut les passer en revue pour nous faire une meilleure idée de l'état de l'art dans le domaine. Nous verrons ainsi comment les travaux de Taylor ont ouvert une aire de production de nombreux modèles. Ainsi, les modèles du risque zéro (Näätänen \& Summala), d'homéostasie du risque (Wilde), d'évitement de la menace (Fuller) et de hiérarchie du risque, ont été les principaux de cette courte période. Par la suite, à un rythme plus lent, certains modèles, comme celui de l'évitement de la menace, ont été repris et modifiés par leurs auteurs, tandis que d'autres ont donné naissance à de nouveaux modèles, comme celui du moniteur de risque. Enfin de nouvelles approches ont cherché à intégrer l'évaluation subjective du risque à la modélisation de la cognition du conducteur, comme dans le cas de la conscience du risque du modèle COSMODRIVE. Nous prenons ici le parti d'examiner ces modèles dans l'ordre chronologique afin de mieux représenter la structure de leur évolution et l'intégration des données empiriques acquises au fil du temps (certains des premiers modèles étant toujours d'actualité). 


\subsection{Les premières conceptions de l'évaluation du risque en conduite automobile}

Les premières considérations de la question du risque dans le domaine de la conduite automobile mettaient l'accent sur le lien entre l'accidentologie et les propriétés intrinsèques des conducteurs, leurs compétences propres. Ainsi, la conduite était considérée comme une activité visuo-motrice et les accidents étaient avant tout envisagés comme étant dus à une défaillance de ces compétences ou à un manque d'habileté [SUM88]. Cette vision des choses a amené à la notion de prédisposition à l'accident comme une sorte de caractéristique individuelle McKenna [MCK82] retrace l'origine de ce terme aux travaux de Greenwood et Woods au début du XXème siècle). Entre les années 50 et 70, les résultats d'études menées dans ce domaine ont invalidé cette conception basée sur les compétences et les aptitudes. Les données d'accidentologie semblaient relativement insensibles à la fois aux actions de formation des conducteurs [ADA70], [PLA71] (issu de Näätänen et Summala [NAT74]) mais également aux améliorations techniques - qu'elles concernent l'infrastructure routière ou les véhicules -. Ce constat, baptisé compensation du risque [COW66], a poussé les chercheurs à tenter de mieux comprendre comment les conducteurs appréhendaient le risque routier.

\subsection{L'étude princeps de Taylor (1964)}

Deux études sont fréquemment reconnues comme fondatrices de l'émergence des théories relatives à l'évaluation subjective du risque dans le domaine de la conduite automobile. Elles ont été menées par Taylor et rapportées dans son article de 1964 [TAY64]. Comme dans le domaine de l'étude de l'évaluation subjective du risque au sens général, Taylor fait le constat d'une différence entre l'évaluation des risques faite par les spécialistes de l'accidentologie et celle des usagers de la route. Les premiers cherchant à avertir le grand public des grands risques d'accidents de la route, les derniers se sentant très libres dans la mesure où, pour la plupart, ils font l'expérience de conduire plusieurs années (ou milliers de kilomètres) sans accident. Taylor fixe alors comme objectif à ces études, d'évaluer l'impact de la variation du risque objectif (dans son amplitude et sa distribution) sur la dimension subjective. Pour appréhender cette dimension subjective, Taylor s'appuie sur des travaux antérieurs tendant à montrer que la conductance cutanée (GSR) des conducteurs est sensible à l'expérience de presque-accidents. Il décide donc de mesurer lors de deux expérimentations s'il existe un lien entre la réponse électrodermale des conducteurs et différentes portions de l'infrastructure routière présentant des niveaux d'accidentologie différents. Les résultats qu'il obtient indiquent selon lui, que la distribution de la conductance cutanée suit et reflète la distribution des valeurs d'accidentologie sur le parcours. L'auteur résume ces premiers résultats ainsi : un observateur extérieur stationnaire voit que là où les virages sont plus rapprochés, les taux d'accidents sont plus élevés, les vitesses sont plus faibles et les événements impactant la GSR sont plus fréquents. Un observateur dans le véhicule avec le conducteur, quant-à-lui, voit les événements impactant la GSR à une fréquence relativement constante indépendamment de la position sur le parcours. Il noterait également que le conducteur tend à passer les virages à un rythme régulier et, s'il est particulièrement observateur, il constatera que le produit de la vitesse moyenne et du taux d'accident est approximativement constant sur le parcours. (P. 447-448). L'auteur note ainsi que les conducteurs semblent maintenir une réponse électrodermale relativement constante tout au long du parcours en adaptant sa vitesse et son rythme de la conduite. Ce résultat majeur constitue une avancée significative dans la démarche de compréhension du phénomène de compensation du risque, aboutissant à un échec relatif des mesures d'amélioration de la sécurité des infrastructures routières. L'intérêt du recours à la GSR doit toutefois être nuancé dans la 
mesure où cet indicateur n'est pas spécifique de l'évaluation subjective du risque.

\subsection{Le modèle du risque zéro (1974)}

Le modèle du risque zéro (ZRT) a été proposé par Näätänen et Summala dans les années 70 [NAA74], [NAA76]. Il s'intéresse à l'explication et à la compréhension du phénomène de compensation du risque. Taylor, dans son étude, proposait déjà l'idée que l'adaptation comportementale à l'origine de la compensation du risque était en lien avec une recherche du conducteur de maintenir un certain niveau d'anxiété ou de stimulation. Le modèle du risque zéro s'inscrit dans ce courant en mettant l'accent sur les aspects motivationnels comme base de comportement et sur son adaptation en fonction du risque perçu. Il est considéré comme l'un des premiers qui soient spécifiquement dédiés à l'évaluation subjective du risque en conduite automobile. Le grand principe de ce modèle est le postulat suivant lequel le conducteur adaptera son comportement de conduite de sorte à rendre nul le risque qu'il évalue subjectivement. La figure 2, extraite de l'article fondateur du modèle [NAA74] présente l'organisation et le fonctionnement du modèle.

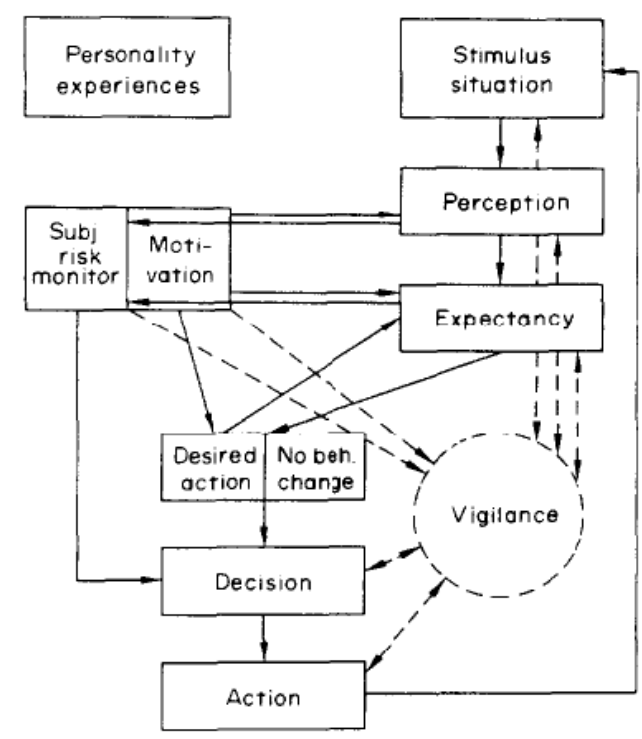

Figure 2. : Modèle du risque zéro [NAA74]

Les modules clés de ce modèle sont :

- Le Module Motivation

- Le Module Moniteur de Risque Subjectif

- Le Module Vigilance

Notons avant tout que le Module Vigilance entretient des interactions avec la plupart des autres modules. La volonté des auteurs est de montrer par ce biais, que chacun des modules composant le modèle est influencé par l'état de vigilance et d'attention du conducteur. Ainsi, par exemple, la motivation comme la situation de conduite, peuvent affecter le niveau de vigilance du conducteur. Commençons à présent la lecture du modèle par l'entrée la plus simple : la situation / stimulus. Elle représente l'environnement extérieur, la situation de conduite. Elle est donc naturellement influencée par les actions préalables du conducteur qui l'ont amené à cet état du monde. Le Module de Perception, comme son nom l'indique, assure la prise d'informations dans cet environnement. Il est la porte d'entrée des stimuli. 
Toutefois, il n'est pas une simple porte passive dans la mesure où il peut être influencé par le Module des Motivations (e.g. un niveau de carburant faible facilitera l'entrée d'informations relatives aux stations-services). Il peut également être influencé par les attentes du conducteur (i.e. Module Expectancy). Nous pouvons constater à ce stade que le Module Motivation agit sur ces deux Modules de Perception et d'Attentes.

Par ailleurs, nous constatons que le Module Motivation agit également sur le Module d'Action souhaitée. C'est ainsi que s'articule le rôle de la motivation dans le modèle. Les auteurs partent du principe que le conducteur présente une motivation à se déplacer dans l'environnement au moyen de son véhicule. Cette motivation va engendrer des actions désirées qui, en temps normal, se traduiront par des décisions (i.e. Module Décision) puis des actions (i.e. Module Action). Ces actions désirées produisent en parallèle, des attentes relatives à la situation de l'environnement. Par exemple, le souhait de vouloir prendre une direction particulière va engendrer l'attente d'indices perceptifs relatifs à cette direction (e.g. panneau de direction). Les actions souhaitées peuvent être de nature variée, comme celle d'accélérer ou de ralentir. Dans certains cas, les indices perceptifs collectés par le Module Perception traduisent un danger potentiel, un risque d'accident. Ainsi, le Module Motivation pourra être la source d'une demande d'augmentation de la vitesse du véhicule afin de réduire le temps de trajet. Toutefois, la présence d'un virage prononcé se profilant à l'horizon, rendra l'accélération dangereuse et le risque de sortie de route important. Les informations relatives à ce virage seront collectées par le Module de Perception et viendront activer le Module Moniteur de Risque subjectif. Ce module constitue une forme de seuil qui, s'il est franchi, court-circuite le Module d'Action Désirée pour directement forcer la décision d'action, de sorte qu'une action soit effectuée pour repasser sous le seuil de risque (e.g. maintenir sa vitesse ou ralentir).

Le fonctionnement en seuil du Module Moniteur de Risque subjectif explique le fait que, selon les auteurs, le conducteur recherche le risque zéro. En effet, selon eux, dès l'instant où les indices perceptifs témoignent d'un risque objectif trop élevé, le Module Moniteur de Risque détectera un risque subjectif non nul et activera la décision de gestion du risque. Les auteurs n'indiquent donc pas que le conducteur ne prend pas objectivement de risque, simplement que si cela se produit, il s'agit d'une erreur involontaire relevant d'une mauvaise évaluation du risque. Tant que ce risque n'est pas subjectivement évalué comme non nul, il n'engendrera pas d'action de réduction du risque. Dans un tel cas le comportement suivra alors les prescriptions faites par le Module Motivation.

Les auteurs se rattachent ainsi aux données fournies par Taylor concernant la réponse électrodermale. Ils tentent de rendre compte du phénomène de compensation du risque en indiquant que les mesures de réduction du risque accidentologique, dans la mesure où elles peuvent laisser l'impression d'une route ou d'un véhicule plus sûrs, repoussent les conditions d'activation du Module Moniteur de Risque subjectif et favorisent donc la satisfaction du Module de Motivation qui, lui, peut souhaiter un temps de trajet toujours plus court.

Dans un article de Summala [SUM88], une précision est apportée concernant le fait que le module n'est pas activé par la présence d'un risque immédiat, mais par l'atteinte d'une marge de sécurité. Ainsi, sont considérés comme activateur du Module Moniteur de Risque Subjectif, les indices perceptifs témoignant d'un non-respect de ces marges. 


\subsection{Le modèle d'homéostasie du risque (1982)}

En 1982 Wilde propose un nouveau modèle, lui aussi inspiré par les résultats des travaux de Taylor [WIL82] mais également par un concept fort connu en physiologie : l'homéostasie. Le modèle d'homéostasie du risque (RHT) représenté dans la figure 3 a été autant populaire que débattu dans la communauté des chercheurs s'intéressant aux questions de l'accidentologie en lien avec l'évaluation subjective du risque.

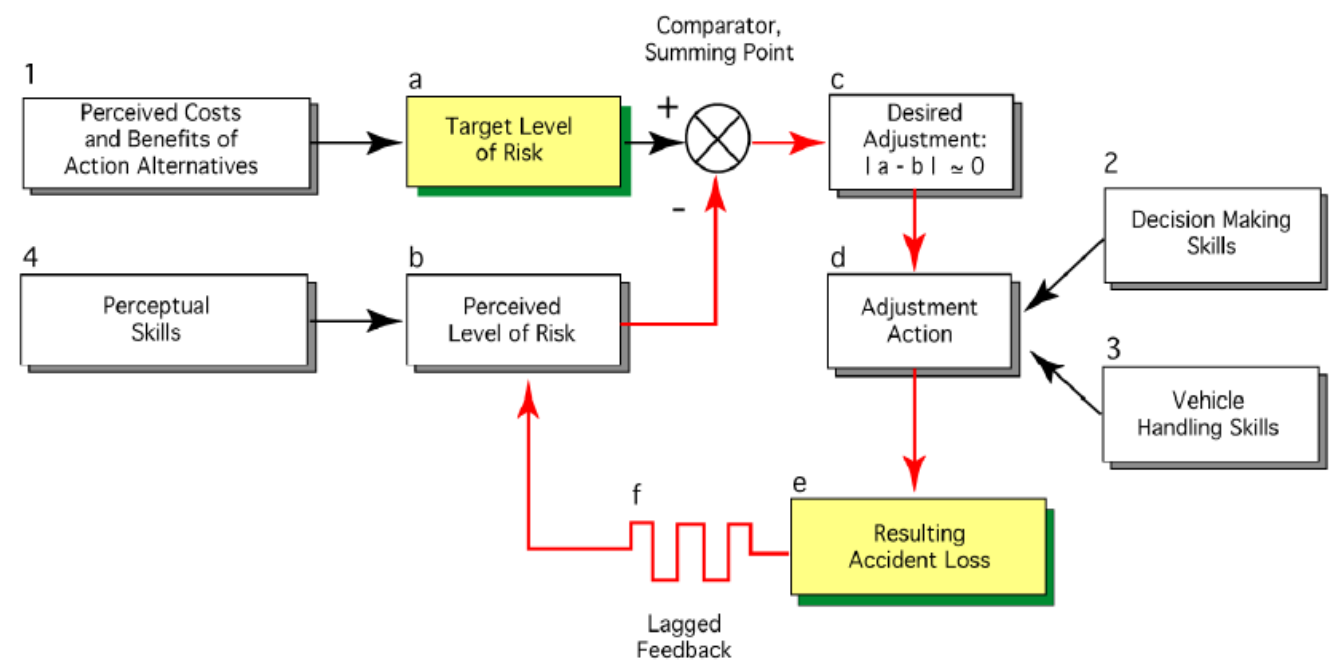

Figure 3. : Modèle d'homéostasie du risque [WIL82]

Le modèle de Wilde postule que le conducteur va déterminer un niveau de risque cible vers lequel il doit tendre et adapter son comportement en conséquence. Pour cela, le conducteur dispose d'un mécanisme de type homéostatique qui lui permet de constamment comparer le niveau de risque subjectif courant au niveau cible. Ce mécanisme, similaire à celui d'un thermostat, permet selon l'auteur de rendre compte du phénomène dit de compensation du risque. La simplicité de ce modèle n'est qu'apparente et pour mieux en saisir la subtilité, il nous faut en explorer les différents aspects plus en détail.

\subsubsection{Le concept d'homéostasie}

Commençons par le concept d'homéostasie. Historiquement découvert par Bernard en physiologie [BER59], puis nommé homéostasie par Cannon en 1929 [CAN29], le concept d'homéostasie a pour but d'expliquer comment un organisme vivant peut maintenir une certaine stabilité de son milieu intérieur. En effet, pour les organismes vivants, quelle que soit leur échelle de taille et d'organisation, il existe des bornes physico-chimiques entre lesquelles le fonctionnement est optimal (i.e. concentrations chimiques, température, etc.). Or, le milieu ambiant est caractérisé par une grande fluctuation des conditions physiques et chimiques.

Un défi important pour la survie et la pérennité, est donc de parvenir à maintenir le milieu intérieur entre les bornes du fonctionnement optimal. Pour cela, l'organisme doit disposer d'au moins trois attributs fondamentaux. Le premier permet de détecter / collecter les changements du milieu intérieur (ou ambiant), le second doit permettre de comparer cette information au niveau cible des paramètres physiques, enfin le dernier doit permettre d'agir sur le milieu intérieur de sorte à minimiser l'écart entre le 
niveau détecté et le niveau cible.

Le principe homéostatique est donc caractérisé par une valeur cible et une suite de mécanismes permettant de faire varier la valeur réelle en direction de cette valeur cible. L'efficacité des mécanismes d'homéostasie est dépendante de la capacité de l'organisme ou du système, à détecter les variations du paramètre considéré, à comparer ces variations à la valeur cible mais également de la capacité des mécanismes de correction à réduire l'écart constaté. Cette efficacité est également affectée par la réactivité de cette chaîne de mécanismes, c'est-à-dire les temps de détection de comparaison et de correction. L'un des intérêts du principe homéostatique réside dans son fonctionnement cyclique, ce qui lui donne une certaine flexibilité et ce qui explique également le fait qu'un mécanisme homéostatique n'assure pas une constance du paramètre qu'il contrôle, mais une oscillation plus ou moins fine de celui-ci autour de la valeur cible. La figure 4, tirée de Wilde [WIL14], montre différents exemples d'oscillations homéostatiques pour une même valeur cible.

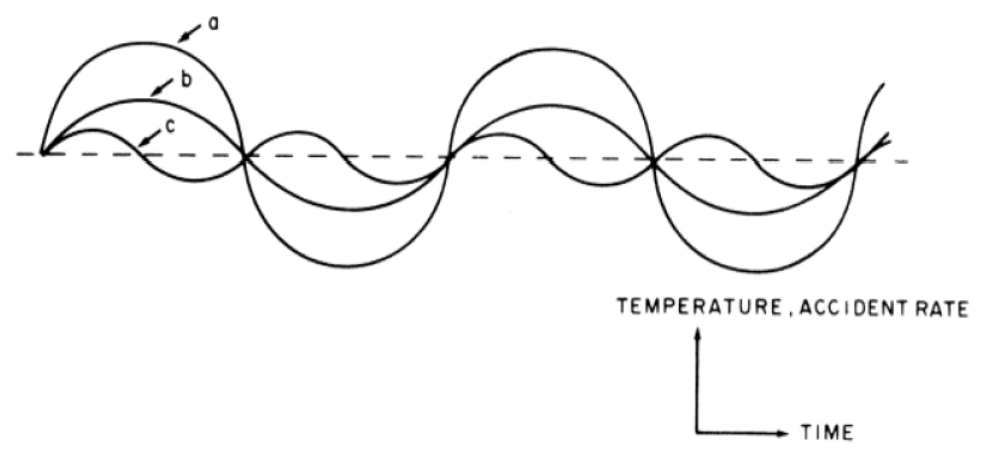

Figure 4. : Exemple d'oscillations homéostatiques [WIL14]

\subsubsection{L'homéostasie appliquée au risque}

Wilde applique donc ce concept de régulation homéostatique à l'évaluation du risque, pour expliquer l'adaptation comportementale des conducteurs aux mesures de sécurité routière et la relative stabilité des données d'accidentologie malgré les mesures prises. Il postule donc que chaque conducteur détermine un niveau de risque cible auquel il comparera la valeur de risque subjectivement évaluée à chaque instant de la situation de conduite. Si le niveau de risque subjectif est inférieur au niveau de risque cible, alors le conducteur modifiera son comportement dans le sens d'une prise de risque (e.g. augmentation de la vitesse). Si le niveau de risque subjectif est supérieur au niveau de risque cible, alors le conducteur adaptera son comportement dans un objectif de réduction de la prise de risque. Enfin, si le niveau de risque subjectif correspond au niveau de risque cible, aucun changement comportemental n'est nécessaire.

Comme pour tout mécanisme homéostatique, l'efficacité de ces processus est affectée par différents éléments. Ici, le niveau de risque subjectivement évalué est dépendant des capacités perceptives et cognitives d'évaluation du risque. En cas de manque de sensibilité de ce système d'évaluation, une valeur erronée sera comparée au niveau de risque cible, ce qui aboutira à un ajustement inapproprié. De même, l'ajustement pourra être inapproprié si le comparateur n'est pas capable de déterminer la différence entre le niveau cible et le niveau de risque évalué, ou encore si les mécanismes décisionnels ou effecteurs sont défaillants. Ainsi, des erreurs de décision concernant l'action ou des capacités de gestion du véhicule altérées, peuvent conduire à une mauvaise adaptation comportementale. Wilde précise que l'estimation 
du niveau de risque par le conducteur à chaque instant est réalisée sur la base de trois sources d'informations :

- L'expérience passée du traffic

- L'estimation du potentiel d'accident de la situation en cours

- Le niveau de confiance du conducteur en ses capacités à gérer la situation et le risque

Sur la base de l'évaluation subjective, le comparateur estime la différence entre cette valeur et la valeur cible. Une inégalité sera détectée si cette différence est supérieure à la différence détectable (i.e. Just Noticable Difference). La différence détectable est simplement la plus petite valeur de différence que le comparateur peut détecter. En dessous de cette valeur, toute différence est indétectable par le comparateur et ne peut donc être prise en compte.

\subsubsection{La notion de risque cible}

Outre ce mécanisme de rétrocontrôle et d'adaptation comportementale, une autre particularité de ce modèle requiert notre attention : la notion de risque cible. En effet, le mécanisme homéostatique permet le maintien du niveau de risque autour d'un niveau cible, ce qui signifie que ce dernier doit être déterminé par le conducteur. Selon l'auteur, le conducteur effectue une comparaison entre les gains attendus (expected gain) et les pertes attendues (expected loss) pour chaque niveau d'exposition au risque (level of exposure to risk), afin de déterminer le bénéfice net attendu (i.e. utilité espérée) de chaque niveau d'exposition au risque. La figure 5 présente un exemple de courbe d'utilité espérée suivant le niveau d'exposition au risque.

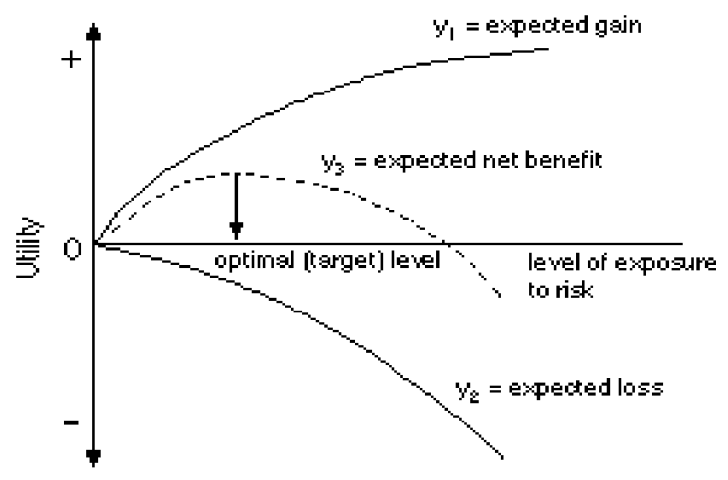

Figure 5. : Courbe d'estimation de l'utilité [WIL14]

Suivant cette procédure, le conducteur détermine le niveau d'utilité (i.e. bénéfice) maximal possible qui correspondra au niveau optimal d'exposition au risque (level of exposure to risk) et sera choisi comme niveau de risque cible. Wilde ne précise pas à quel point ce raisonnement est rationnel chez le conducteur (cf. théorie de l'utilité subjective espérée). Toutefois, il indique qu'il effectue ses estimations sur la base des données accidentologiques qu'il connaît, ainsi que d'autres éléments plus subjectifs. Quoi qu'il en soit, le conducteur établit un niveau de risque cible non nul, correspondant au niveau optimal d'utilité qu'il a estimé. Le modèle d'homéostasie du risque est donc motivationnel par essence, dans la mesure où le comportement est motivé par la réduction de l'écart entre le risque courant subjectivement estimé et le risque cible (lui-même déterminé par une estimation subjective d'utilité). 
Ce double mécanisme a plusieurs conséquences sur la manière d'interpréter et de prédire les comportements et le risque effectivement pris par les conducteurs. Premièrement, il permet, contrairement au modèle de Näätänen et Summala [NAA76], d'imaginer un conducteur qui prendrait des risques volontairement. Deuxièmement, il exclut la recherche d'un risque nul, ce qui est à l'opposé de la proposition avancée par la théorie du risque zéro. La conception de Wilde propose que le risque puisse être subjectivement évalué comme non nul, sans pour autant que cela constitue une barrière à l'acceptation de celui-ci et donc une modification du comportement dans le sens de la réduction du risque pris. Elle autorise donc une prise de risque intentionnelle et consciente. Troisièmement, le modèle de Wilde, par sa constitution, fait reposer les comportements de prise ou d'évitement de risque sur l'estimation de l'utilité espérée. Ceci a pour conséquence de retirer beaucoup d'importance à l'estimation subjective du risque dans la mesure où, quelle qu'elle soit, elle n'est pas responsable directement des choix comportementaux. Ainsi, selon Wilde, la manière la plus efficace de diminuer la prise de risque effective, n'est pas d'apporter des améliorations technologiques ou perceptives afin d'améliorer les performances des conducteurs à évaluer le risque ou à le gérer, mais d'agir au niveau de la procédure d'estimation de l'utilité subjective espérée, en jouant sur le ratio entre les bénéfices et les pertes associées à la prise de risque. Ainsi, Wilde identifie quatre voies pour modifier le seuil de risque cible dans le but de le faire baisser:

- Diminuer les bénéfices associés à la prise de risques

- Augmenter les pertes associées à la prise de risques

- Augmenter les bénéfices associés à une conduite prudente

- Diminuer les pertes associées à une conduite prudente

Selon lui, les échecs des politiques de sécurité routière sont donc à rechercher dans ce mécanisme qui permet de déplacer le niveau de risque cible vers le bas. Ainsi, si une mesure de sécurité routière ne cherche pas à faire baisser l'utilité subjective espérée d'une prise de risque, elle sera inefficace car compensée comportementalement.

\subsubsection{Arguments et données empiriques}

Pour justifier son approche, Wilde s'appuie sur différents types de résultats, certains impliquant un déplacement du risque cible et d'autres non.

\subsubsection{Le changement de sens de circulation Suédois}

Wilde rapporte le cas très célèbre du changement de côté de conduite en Suède à la fin des années 60 [WIL82]. À cette époque, les autorités suédoises ont pris la décision de passer du jour au lendemain, d'une conduite à gauche à une conduite à droite. Nous pourrions nous attendre à ce qu'une telle mesure aboutisse dans les premiers temps à une augmentation significative de l'accidentologie, or l'effet fût totalement contraire. L'interprétation fournie par la théorie de l'homéostasie du risque est que cette décision de changement de sens de circulation a considérablement élevé l'estimation du niveau de risque accidentologique associé à cette réforme. Les conducteurs ont donc surévalué le risque subjectif de chaque situation de conduite. Cette évaluation subjective biaisée s'est alors trouvée à un niveau bien supérieur au risque cible habituel, une telle différence était donc homéostatiquement compensée par une conduite bien plus prudente. Un changement comportemental qui s'est, au final, traduit par une baisse de $17 \%$ 
de la mortalité routière dans l'année suivant l'introduction de cette nouvelle mesure. Par la suite, les conducteurs ont petit à petit pu faire l'expérience d'un risque moins élevé qu'initialement supposé ce qui, associé à l'apprentissage de la nouvelle façon de conduire, à progressivement diminué l'écart entre le risque subjectif et le risque cible. Cette adaptation s'est alors traduite par une remontée progressive du niveau d'accidents aux valeurs initiales.

Cette première donnée est un cas relativement particulier dans la mesure où c'est l'évaluation subjective du risque qui a été momentanément biaisée, et non le niveau de risque cible qui a été modifié. Selon Wilde, le fait que le niveau d'accidentologie soit remonté à sa valeur initiale est un argument en faveur de sa conception selon laquelle une baisse durable de la mortalité routière ne peut être obtenue si l'on ne cherche pas à faire baisser le niveau de risque cible.

\subsubsection{L'expérience des taxis munichois}

Afin de tester la théorie de l'homéostasie du risque, et en particulier les effets d'une mesure de type amélioration technologique, une expérimentation a été conduite au début des années 90, impliquant des conducteurs de taxi à Munich [WIL14], [ASC87]. Dans cette étude les chauffeurs de taxis ont été aléatoirement affectés dans deux groupes. Les conducteurs du premier groupe ont reçu des véhicules équipés d'un système de freinage ABS tandis que les conducteurs du second groupe ont reçu des véhicules en tout point identique à l'exception du système de freinage qui était traditionnel. À l'issue de la première phase de l'étude, les valeurs d'accidentologie des deux groupes ont été comparées et n'ont révélé aucune différence. Ce résultat tend à montrer que l'amélioration technique que représente l'ABS ne s'est pas traduite par une baisse de l'accidentologie. Pour mieux comprendre ces résultats, un examen des données supplémentaires acquises pendant l'étude a montré que les conducteurs du groupe des taxis avec ABS avaient une conduite significativement moins précise et plus brutale, avec des accélérations et décélérations plus fortes que les conducteurs ayant des taxis équipés de freins traditionnels. Un tel résultat traduit l'adaptation comportementale et est conforme aux prédictions de Wilde dans la mesure où la simple introduction de cette amélioration technique n'aboutissait pas à l'objectif de faire diminuer chez les conducteurs le niveau de risque cible visé.

Dans une extension de l'étude la compagnie de taxi a introduit une mesure supplémentaire consistant à faire payer une partie des réparations par les chauffeurs de taxis accidentés (ils étaient également avertis de la possibilité d'être licenciés en cas d'accidents trop nombreux). L'auteur rapporte qu'au cours de cette seconde phase de l'étude, le nombre d'accidents a considérablement chuté. Ce résultat est là encore argument en faveur de la théorie de l'homéostasie du risque puisque cette nouvelle mesure visait uniquement à modifier le rapport des gains et des pertes associées à l'occurrence d'un accident. Autrement dit, à modifier le niveau d'utilité espérée associée à la prise de risque. Il est donc possible de supposer que face à ce changement du rapport bénéfices / pertes, les conducteurs aient abaissé leur niveau de risque cible et adapté leur comportement quel que soit le type de taxi utilisé (i.e. avec ou sans ABS).

Cette étude en deux temps, présente l'intérêt d'avoir cherché à évaluer dans un premier temps les effets d'une action purement technologique sans incidence sur le niveau de risque cible puis, dans les mêmes conditions, les effets de l'introduction d'une action spécifiquement destinée au déplacement du risque cible. La stratégie adoptée dans cette étude était d'augmenter le coût en cas d'accident. Si elle a fonctionné dans ce cas, Wilde indique cependant qu'elle n'est pas forcément la plus efficace et qu'elle 
présente des limites. En effet cette stratégie de type punitive permet d'obtenir des effets jusqu'à une certaine limite. L'auteur évoque ainsi de nombreux travaux témoignant de l'existence d'une telle limite au-delà de laquelle les sujets refusent de se soumettre à la punition, parce qu'ils l'estiment injuste et disproportionnée. Cet effet plafond se traduit alors par l'apparition de plusieurs phénomènes tels que : la stagnation de l'effet positif, la tendance au contournement de la règle, et enfin la réactance aussi appelée retour de flamme, et qui se traduit par un comportement volontairement orienté vers la transgression et la prise de risque.

\subsubsection{Les données issues de mesures de récompense}

Si la stratégie consistant à augmenter les pertes associées à la prise de risque s'avère d'une efficacité limitée, Wilde indique qu'un nombre conséquent d'études démontrent l'intérêt des mesures consistant à augmenter le bénéfice associé à un comportement prudent. Nous noterons le cas d'une telle disposition mise en place par une grande entreprise du secteur agro-alimentaire qui a pris la décision d'introduire en 1957, une prime à ses 600 salariés conducteurs poids-lourd pour chaque semestre sans accident responsable. Les résultats se sont avérés extrêmement positifs et durables. En effet, le taux d'accident responsable est descendu d'un tiers la première année, puis a progressivement poursuivi sa descente jusqu'à atteindre $14 \%$ de sa valeur initiale après une vingtaine d'années d'application de la disposition. Un autre résultat intéressant issu de ce rapport concerne celui des accidents non responsables (donc non concernés par la prime) qui a lui aussi chuté pour atteindre, en 1981, $25 \%$ de son niveau en 1956.

Le modèle de l'homéostasie du risque insiste donc sur deux aspects important de l'évaluation et de la prise de risque : la notion de risque cible autorisant la prise de risque volontaire et le recours au mécanisme d'homéostasie permettant de réguler le comportement autour de la valeur de risque cible.

\subsection{Le modèle d'évitement de la menace (1984)}

À partir de 1984, Fuller va proposer une suite de modèles d'évaluation subjective du risque en conduite automobile, le premier étant le modèle d'évitement de la menace [FUL84]. Situé dans la même ligne que le modèle du risque zéro [NAA76], le modèle d'évitement de la menace suppose qu'une des motivations fortes du conducteur pendant l'activité est d'éviter les risques. Pour cela, Fuller reprend les concepts de conditionnement (classiques et opérants) issus du behaviorisme comme base explicative de l'apprentissage, du comportement et de l'estimation du risque. Selon Fuller, le conducteur serait à la recherche d'indices perceptifs témoignant d'une possibilité d'accident ou de la survenue d'un danger. Dans les cas où de tels indices sont présents, le conducteur procédera à un ajustement comportemental de type évitement de la menace. Le processus d'apprentissage consiste simplement en un mécanisme de récompense ou de punition permettant le renforcement ou l'oubli des comportements sur la base des indices perceptifs relevés. Ce mécanisme d'apprentissage n'est cependant pas considéré par Fuller comme la seule et unique voie d'apprentissage, dans la mesure où les indices perceptifs ne doivent pas nécessairement avoir déjà été observés et vécus par le conducteur pour être mémorisés. La figure 6 présente l'organisation du modèle de Fuller

Au travers de ce modèle, l'objectif de Fuller est de parvenir à gérer un certain nombre de cas qui ne le sont pas dans le modèle du risque zéro, en particulier celui de la prise volontaire de risque. En 


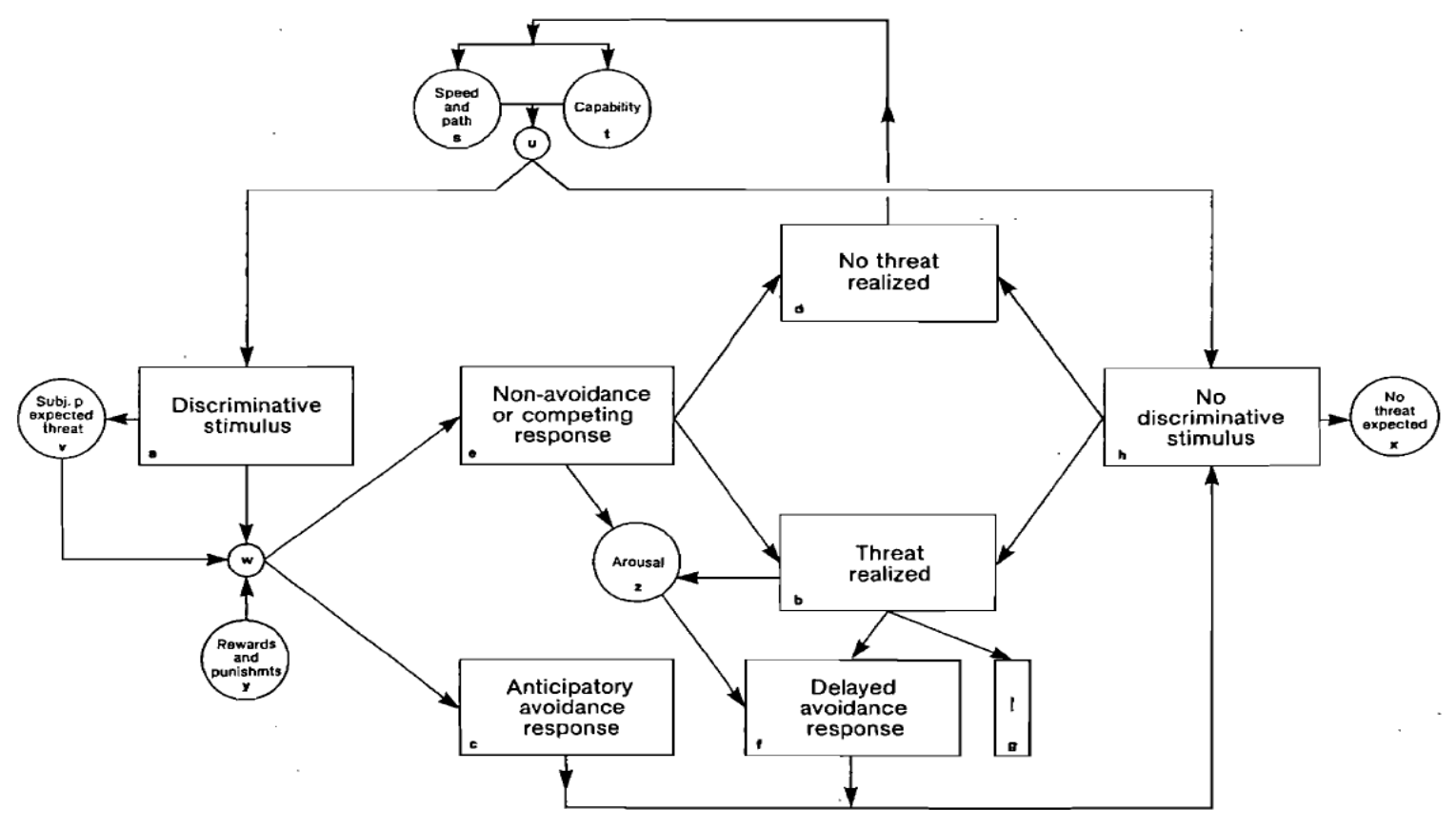

Figure 6. : Modèle d'évitement de la menace [FUL84]

effet, le modèle d'évitement de la menace prévoit l'existence d'une réponse dite concurrente (competing response). Il s'agit en fait, d'une alternative comportementale plus risquée, mais permettant possiblement d'obtenir une certaine récompense (via un gain de temps ou un niveau d'excitation plus important). Fuller cherche aussi à gérer par son approche, le problème de la rareté statistique des événements associés au risque (i.e. accidents). Ceci explique qu'il propose de ne pas évaluer ces événements en eux-mêmes, mais les éventuels indices précurseurs de ceux-ci, indices que Fuller appelle discriminative stimulus.

Si nous reprenons le modèle dans l'ordre, nous pouvons partir d'un contexte simple impliquant un conducteur pourvu de capacités données dans une situation donnée. Un stimulus discriminant peut alors être présent dans cette situation (e.g. virage à faible visibilité). S’il est détecté, les expériences passées, les objectifs et les compétences estimées du conducteur vont être pris en compte afin de déterminer si une action d'évitement anticipée du risque (i.e. anticipatory avoidance response) doit être mise en place, ou si l'évitement du danger peut être repoussé au profit d'une réponse concurrente.

Dans le cas d'un choix de réponse d'évitement anticipé, deux cas peuvent se produire. Si la menace se concrétise, alors le comportement d'évitement anticipé sera récompensé. Dans le cas contraire, il ne le sera pas, ce qui aura pour conséquence, une diminution de la probabilité de reproduction de l'évitement anticipé lors de la prochaine détection d'un stimulus discriminant similaire.

Dans le cas du choix d'une réponse concurrente ou d'un report du comportement d'évitement (i.e. non-avoidance response), le conducteur poursuivra son évolution dans la situation de conduite sans modification ou exécutera la réponse concurrente choisie (e.g. accélérer, dépasser, etc.). Ce choix aura pour conséquence une augmentation du niveau d'excitation physiologique (i.e. arousal). La situation, de son côté, va évoluer et voir la menace se réaliser ou non. En cas de non réalisation, le choix du report sera renforcé. En cas de survenue du danger, un évitement plus brutal (i.e. delayed avoidance response) devra être mis en place, ou bien un accident se produira. Dans les deux cas, il s'agira d'une forme de punition dont la conséquence sera une diminution de la probabilité du choix de report lors de la prochaine détection du stimulus discriminant. Fuller prévoit également le cas où aucun stimulus discriminant n'est 
détecté, soit parce qu'il n'existe pas, soit parce que le conducteur ne le reconnaît pas comme tel. Dans ce cas, tout se passe comme dans celui précédemment évoqué du choix d'un report de l'évitement. L'auteur prévoit enfin, un cas d'évitement anticipé partiel auquel le conducteur aura recours en cas d'incertitude trop forte concernant le stimulus discriminant.

Bien qu'étant principalement dans la veine du modèle du risque zéro, Fuller précise que le modèle d'évitement de la menace peut également rendre compte des phénomènes d'adaptation comportementale au même titre que le modèle d'homéostasie du risque. Pour cela, il évoque les cas de manifestation rare de la menace. Nous pouvons par exemple imaginer des situations de conduite où des signes précurseurs ne sont pas d'une grande fiabilité, c'est-à-dire qu'ils sont relativement rarement suivis de la survenue du danger. Dans ces cas, le comportement d'évitement anticipé va progressivement s'éteindre jusqu'à la prochaine survenue du danger. Ce cas rare va alors pousser l'évitement anticipé à réapparaître dans des situations similaires avant une nouvelle extinction progressive. Dans cette situation, nous avons donc également affaire à un phénomène cyclique.

Nous devons ici, noter que ni les règles précises de renforcement et de punition, ni les conditions à partir desquelles un évitement est considéré comme anticipé ou reporté ne sont explicitées pour ce modèle. Bien que Fuller indique qu'un évitement reporté (delayed response) correspond à une manœuvre d'évitement d'urgence, il est néanmoins, contraint d'évoquer le cas des évitements partiels qui révèlent probablement, de potentielles lacunes du modèle dans la gestion de la temporalité et de l'incertitude. Fuller proposera par la suite une évolution de ce modèle que nous aborderons plus loin dans cet article.

\subsection{Le modèle hiérarchique du risque (1988)}

Van Der Molen et Bötticher, dans un article de 1988, rapportent avoir testé chacun des trois précédents modèles, sur un exemple simple [VAN88]. Ils choisissent la situation de conduite suivante : un conducteur doit décider ou non de dépasser un poids lourd sur une route à double sens avec des véhicules arrivant en face tout en ayant pour objectif général de maintenir une vitesse globale qu'il a déterminée afin d'être à l'heure à un concert. Le point clé de cette situation est donc la décision d'effectuer ou non le dépassement, ce qui implique que le conducteur se livre à une évaluation subjective du risque associé à cette situation pour prendre sa décision. À titre de précision, les auteurs indiquent les postulats qu'ils fixent pour tous les modèles afin de rendre leurs résultats comparables. Ils présument ainsi que le conducteur est capable d'étudier et d'estimer toutes les probabilités et utilités subjectives pertinentes. De même, ils supposent que le conducteur choisira rationnellement l'alternative ayant l'utilité subjective la plus élevée. Pour déterminer cette solution, les auteurs se réfèrent à une formule de l'utilité subjective espérée issue de la littérature [EDW54]. Les auteurs indiquent enfin qu'ils ne prétendent pas à la validité de ce test au sens psychologique, mais que celui-ci permet néanmoins de révéler certaines lacunes et contradictions dans les modèles testés. Ainsi, par exemple, ils indiquent que plusieurs de ces modèles souffrent d'un manque de spécifications, rendant hasardeuse ou impossible leur utilisation sur des données réelles. Ce constat les amène à proposer leur propre modèle hiérarchique du risque. La particularité de leur modèle est d'intriquer les processus d'évaluation du risque aux strates cognitives déjà admises dans les modèles de l'activité du conducteur (i.e. [MIC85]). La figure 7 présente l'organisation du modèle.

Ils reprennent ainsi les trois principaux niveaux hiérarchiques proposés par Michon que sont : le niveau stratégique, le niveau tactique et le niveau opérationnel [MIC85]. Nous retrouvons donc les aspects très 


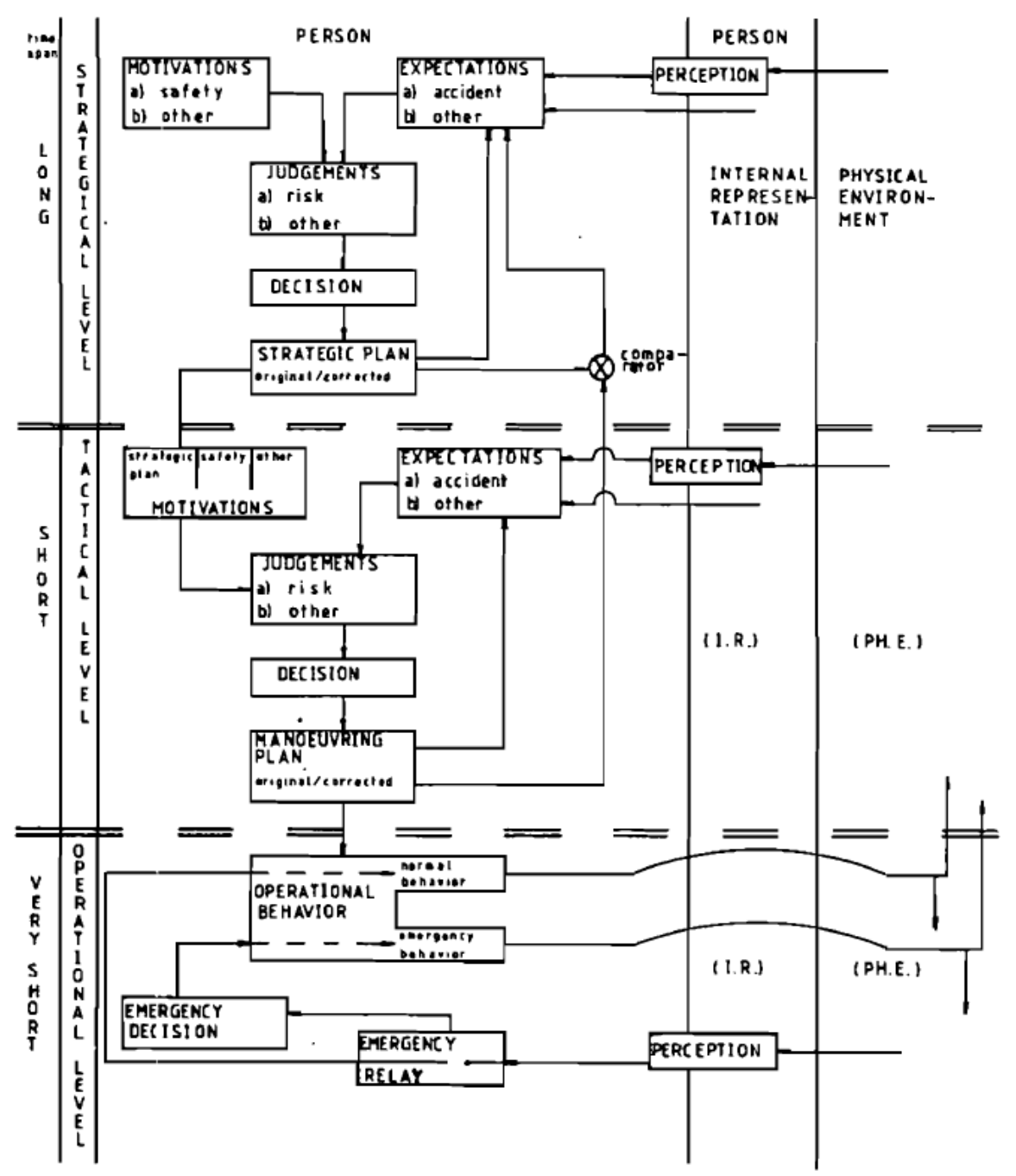

Figure 7. : Modèle hiérarchique du risque [VAN88] 
généraux de la planification du trajet (i.e. itinéraire général, gestion du trafic, des contraintes horaires) à effectuer au niveau stratégique, les aspects relatifs à l'analyse et à la planification de la gestion de la situation de conduite en cours au niveau tactique (i.e. décisions locales de changement de voie, de dépassement, de suivi d'itinéraire, etc.) et enfin les aspects de très bas niveau relatifs à l'exécution et au contrôle du véhicule au niveau opérationnel (i.e. actions sur le volant et les pédales).

La particularité de ce modèle est donc que, pour chacun des niveaux hiérarchiques que nous venons d'évoquer, les auteurs ont inclus des modules spécifiquement dédiés à l'évaluation du risque et à sa gestion. Ainsi, au niveau stratégique, les auteurs placent un module d'évaluation (i.e. module jugements) qui est alimenté par les motivations d'un côté, et les attentes (i.e. expectations) de l'autre. Les auteurs font la distinction entre les motivations relatives à la sécurité routière et les autres motivations. De même, ils distinguent les attentes relatives aux accidents des autres types d'attentes. Le module de jugement du niveau stratégique effectue au moins deux types de jugements : les jugements relatifs au risque et les autres. Ces jugements sont utilisés pour prendre une décision stratégique qui sera formulée au travers d'un plan stratégique. Ce plan stratégique tiendra donc compte à la fois des aspects relatifs au risque et des autres aspects associés au trajet à effectuer en voiture (i.e. contraintes temporelles, itinéraires connus et préférés, etc.). Notons au passage que ce plan stratégique peut être corrigé à tout instant si des informations (notamment perceptives) viennent contredire les attentes stratégiques. À ce niveau hiérarchique, l'échelle temporelle est relativement longue, et généralement, les temps de décisions le sont également.

Une fois le plan stratégique établi, nous pouvons porter notre intérêt sur son impact au niveau inférieur : le niveau tactique. Ici, les modules spécifiquement dédiés aux processus d'estimation du risque sont structurés de manière similaire. Un module d'attente est approvisionné par les informations perceptives et gère les attentes relatives aux accidents potentiels et les autres attentes. Ce qui le distingue du module de même nom de niveau hiérarchique supérieur, ce sont les types d'attentes qui s'y trouvent. Ainsi, au niveau stratégique, les attentes relatives aux accidents seront très générales (e.g. ce tronçon routier est fréquemment verglacé), tandis qu' au niveau tactique, les attentes relatives aux accidents seront contextualisées à la situation particulière (e.g. ce véhicule à un comportement dangereux, un accident est possible). Au niveau tactique, le module de motivations comporte trois catégories de motivations : les motivations relatives à la sécurité, le plan stratégique et les autres motivations. Le plan stratégique représente donc une forme particulière de motivation dont le module de jugement tactique tiendra compte, en plus des autres motivations identifiées. Là encore, le jugement pourra porter sur le risque ou sur d'autres aspects tactiques de la situation de conduite. Ce jugement permettra la prise de décisions tactiques, aboutissant à un plan de manœuvre (nouveau ou correctif). À ce niveau hiérarchique, l'échelle temporelle est beaucoup plus courte (i.e. de quelques secondes à quelques dizaines de secondes).

Une fois le plan de manœuvre déterminé, il est transmis au niveau hiérarchique inférieur : le niveau opérationnel. Ce niveau est celui de l'action et du très court terme où le plan de manœuvre est exécuté et traduit en instructions motrices. Les auteurs intègrent également ici, une composante relative à l'évaluation subjective du risque. Toutefois, ce niveau hiérarchique étant très bas, l'élaboration des traitements relatifs au risque ne peut être aussi importante que pour les niveaux supérieurs. Les auteurs distinguent donc deux types de cas de figure :

- La conduite normale, dans laquelle le risque est géré par les niveaux supérieurs;

- La conduite d'urgence, dans laquelle une information perceptive a indiqué un risque imminent. 
Le cas de la conduite d'urgence est particulier, dans la mesure où elle fait intervenir un module de relais d'urgence (i.e. emergency relay) et un module de décision d'urgence. Ces modules servent de courtcircuit permettant l'implémentation immédiate d'une manœuvre d'urgence stéréotypée (e.g. freinage d'urgence).

En composant ainsi leur modèle, les auteurs cherchent à assembler et compléter les trois précédents, tout en les intégrant de manière plus fine à un modèle cognitif de l'activité de conduite. En ce sens, ce modèle apporte une spécification plus précise, et cherche à mieux rendre compte de la diversité des cas de figure possibles. Il permet en outre, de mieux expliciter les différents niveaux hiérarchiques au sein desquels peut prendre place l'évaluation subjective du risque. En effet, ce n'est probablement pas la même chose d'évaluer le risque pour déterminer son itinéraire que d'évaluer le risque de collision dans la prochaine seconde. Les auteurs cherchent également à maintenir la dimension motivationnelle, si importante dans le cadre de l'évaluation subjective du risque. Van Der Molen et Bötticher vont plus loin en cherchant à indiquer à quel niveau certains concepts développés dans les autres modèles peuvent s'appliquer (i.e. le moniteur de risque subjectif de Näätänen et Summala est évoqué comme pouvant être candidat au module de décision d'urgence). Enfin, dans leur article, les auteurs indiquent que le modèle peut encore être amélioré, notamment en incluant les cas d'erreurs tels que conceptualisés par Reason [REA90].

\subsection{Le modèle d'allostasie du risque (2000 - 2011)}

Dans les années 2000, Fuller a fait évoluer son approche de l'évaluation et de la gestion du risque par évitement de la menace en proposant, tout d'abord, le modèle d'interface tâche - capacité, puis ensuite une version plus aboutie de ses conceptions sous le nom de modèle d'allostasie du risque.

\subsubsection{Le modèle d'interface tâche - capacité (2000)}

Le modèle de d'interface tâche - capacité (ITC) de Fuller, présenté en figure 8, met l'accent sur le caractère autorégulé de l'activité de conduite [FUL00]. Poursuivant son approche de l'évitement de la menace, Fuller aborde la question sous un angle nouveau en remettant en cause l'idée que le risque subjectif évalué soit directement associé à la probabilité d'accident. Selon lui, le conducteur n'effectue pas d'estimations de la probabilité d'accident, mais une gestion de la tâche de conduite. Pour expliquer son approche, il avance le fait que les accidents sont la conséquence d'une perte de contrôle de la situation à un instant donné. En d'autre termes, un accident se produit lorsque les capacités du conducteur à exécuter la tâche de conduite, sont dépassées par les demandes de la situation. Il propose donc l'existence d'une interface entre les capacités du conducteur et les demandes de la situation de conduite. Tant que les demandes de la situation sont inférieures ou égales aux capacités, la situation est contrôlable et contrôlée. Si les demandes dépassent les capacités, alors le contrôle de la situation est perdu et, à moins d'une action compensatoire des autres acteurs impliqués (ou d'une certaine tolérance à l'erreur de la part de l'environnement), l'accident se produit. Suivant ce modèle, pour un conducteur, le risque subjectif ne serait pas le risque d'un accident mais le risque de la perte de contrôle de la situation. Cette évaluation du risque serait donc très étroitement liée à la difficulté d'exécution de la tâche de conduite. De nombreux facteurs interviennent dans l'établissement de cette interface. Comme la figure suivante le montre, les capacités du conducteur sont sous l'influence de facteurs permanents (i.e. Training, education, experience, com- 
petence) et sous l'influence de facteurs plus transitoires (i.e. human factors, attention, vigilance, etc.). De l'autre côté, les demandes imposées par la situation de conduite sont également sous l'influence de plusieurs facteurs liés notamment à l'environnement de conduite, aux autres usagers de la route et au véhicule en lui-même. L'adéquation entre ces contraintes de capacités et d'exigences de la situation, est gérée, dans la mesure du possible, par le conducteur, au travers de ses actions sur la vitesse et la trajectoire du véhicule, ou encore de ses interactions avec les autres acteurs impliqués.

Selon ce modèle, l'évaluation subjective du risque et sa gestion se font grâce à l'évaluation de la probabilité de perdre le contrôle de la situation, et donc de la difficulté éventuelle à faire correspondre les capacités de contrôle du conducteur et les demandes de la tâche de conduite.

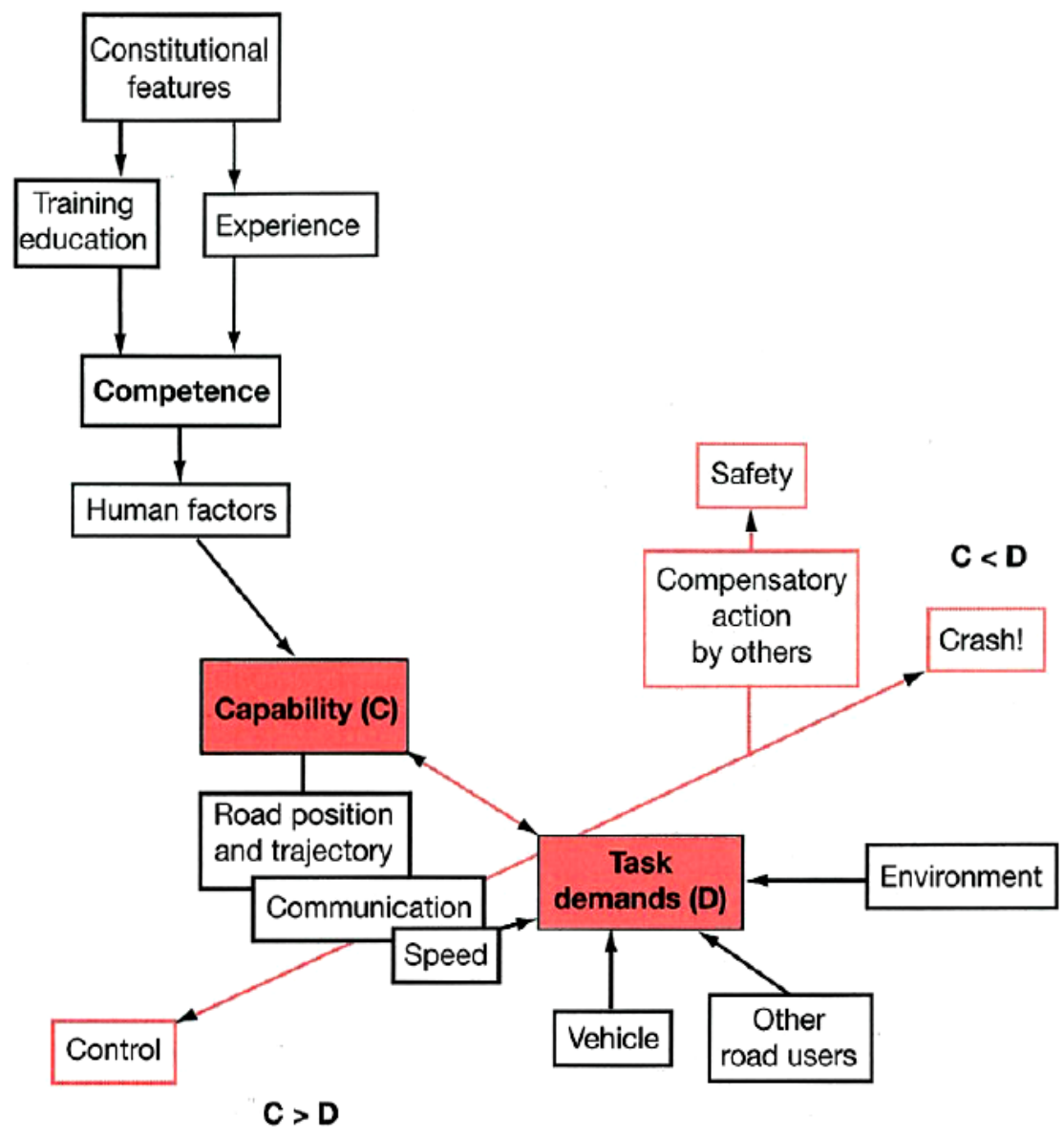

Figure 8. : Modèle d'interface Tâche - Capacité [FUL00]

\subsubsection{La théorie de l'allostasie du risque (2008)}

Quelques années plus tard, Fuller propose une version complétée du modèle d'interface Tâche - Capacité : la théorie de l'allostasie du risque. Il commence en 2005 [FUL05] par ajouter un mécanisme de régulation de la difficulté de la tâche de conduite en s'inspirant de celui proposé par Wilde. Il s'agit donc du mécanisme d'homéostasie de la difficulté de la tâche, dont la figure suivante présente le fonctionnement. Ce thermostat de la difficulté de la tâche, présenté en figure 9 permettrait au conducteur de déterminer le seuil de difficulté cible qu'il souhaite accepter. Pour justifier cette substitution de la difficulté de la tâche de conduite à l'évaluation subjective probabiliste du risque, Fuller produit des résultats issus d'une expérience dans laquelle des vidéos de conduite à différentes vitesses (filmées du point de 
vue du conducteur) sont présentées aux participants [FUL08]. Les participants devaient ensuite estimer la difficulté de la tâche de conduite, le risque subjectif et le risque d'accident. Les données montrent une très forte corrélation entre les estimations de risque subjectif et les estimations de difficulté ( $r=.97)$, les deux dimensions croissent de manière similaires en suivant l'augmentation de la vitesse. Les estimations du risque d'accident, quant à elles, ne suivent pas exactement la même tendance dans la mesures où, pour les vitesses faibles, elles sont évaluées comme nulles jusqu'à un certain point avant de commencer à monter. Dans une expérience complémentaire, reposant sur les mêmes principes (i.e. évaluation de situations de conduite en vidéo), il a été demandé aux participants quelle était la vitesse à laquelle la conduite était la plus confortable selon eux. La plupart d'entre eux choisirent une vitesse inférieure à la vitesse seuil à partir de laquelle ils estimaient que le risque d'accident comme supérieur à zéro. Ces résultats doivent toutefois être considérés avec précaution dans la mesure où le modèle de Fuller postule un mécanisme homéostatique de régulation de la difficulté de la tâche de conduite or, l'expérimentation n'impliquait pas une tâche de conduite mais le visionnage d'une séquence de conduite. Fuller avance, dans ces réflexions additionnelles, que l'homéostasie du risque de Wilde pourrait être vue comme un cas particulier de l'homéostasie de la difficulté de la tâche, dans la mesure où les données semblent indiquer que la probabilité d'accident n'est corrélée à l'estimation subjective du risque qu'à partir d'un certain seuil.

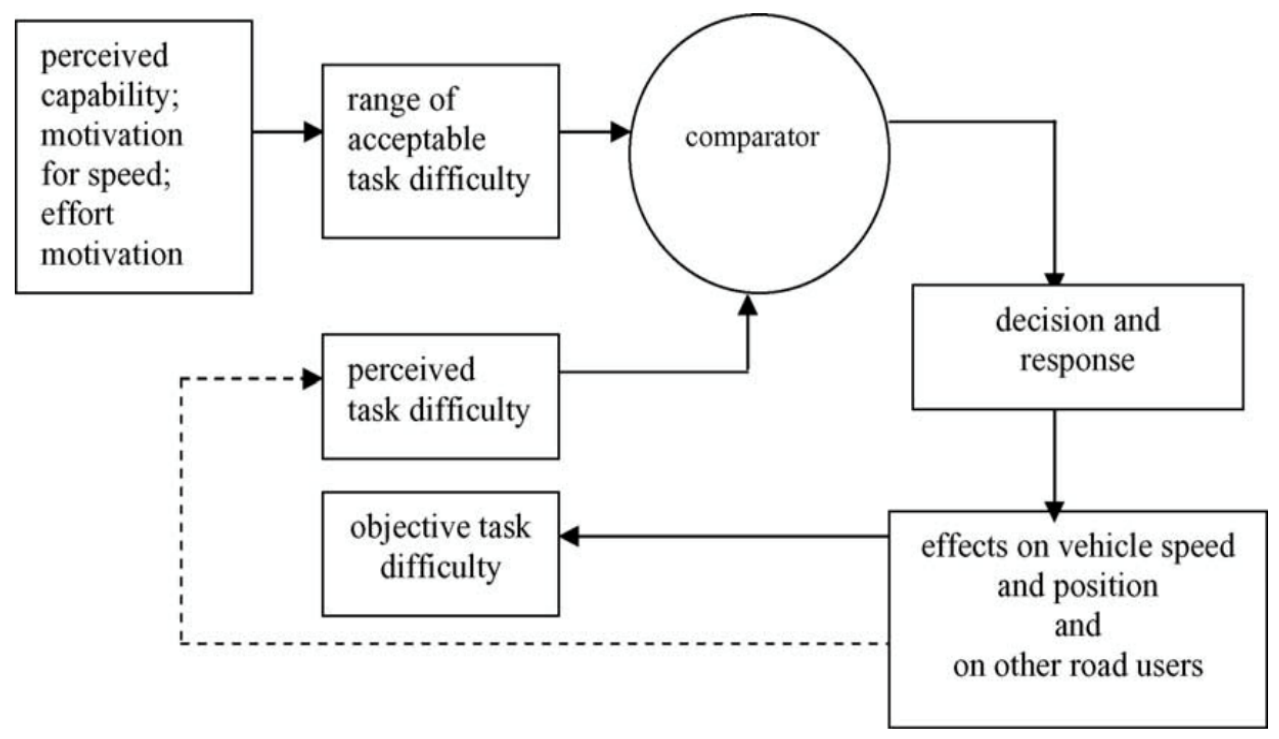

Figure 9. : Mécanisme d'homéostasie de la difficulté de la tâche [FUL05]

L'identification du lien entre l'évaluation subjective de la difficulté et celle du risque, pousse Fuller à adapter son modèle combinant l'interface tâche - capacité et le mécanisme d'homéostasie de la difficulté, pour y intégrer un mécanisme de monitorage constant du risque comme un reflet de la difficulté de la tâche de conduite. Selon cette nouvelle conception, qu'il baptise allostasie du risque, le conducteur dispose d'une plage de sensation de risque préférentielle. Cette sensation de risque serait en fait, le reflet du niveau de difficulté de la tâche de conduite. Elle servirait de moniteur pour que le conducteur puisse gérer la difficulté de la tâche de conduite [LEW09]. La figure 10 présente le modèle issu de [FUL11].

Comme pour le modèle du moniteur de risque de Vaa que nous développerons dans la section suivante, Fuller justifie son modèle en faisant référence à la théorie des marqueurs somatiques de Damasio [DAM94] ainsi qu'aux travaux de Slovic concernant l'heuristique de l'affect [SLO04], [SLO07]. Le modèle de l'allostasie du risque accorde donc une place primordiale aux aspects émotionnels, dans 


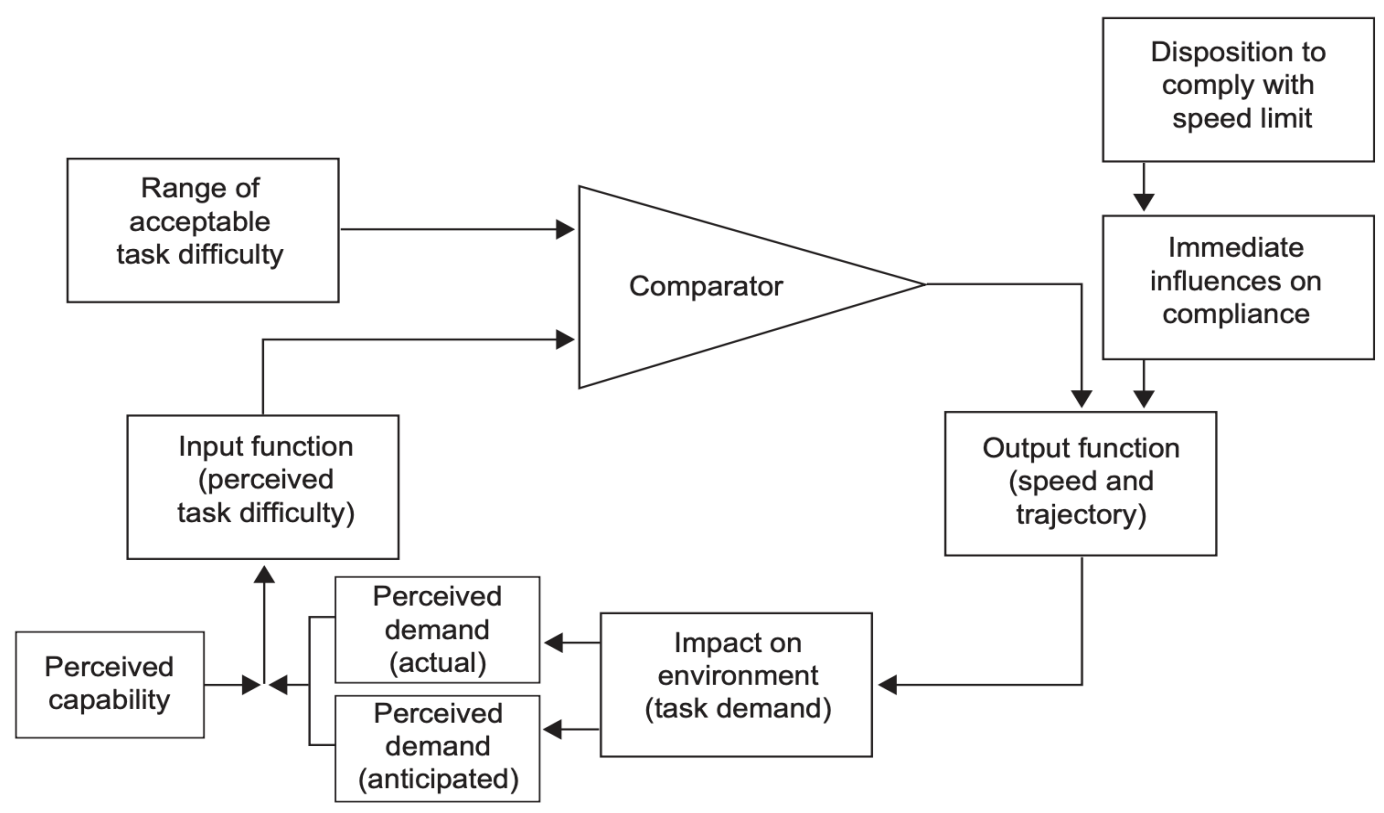

Figure 10. : Modèle d'allostasie du risque [FUL11]

l'évaluation du risque et la prise de décision en conduite automobile.

\subsection{Le modèle du moniteur de risque (2007)}

Durant cette même période, Vaa fait un bilan des modèles de l'évaluation du risque en conduite automobile [VAA01], [VAA14]. Selon lui, la théorie de Wilde présente plusieurs défauts qui ont égaré les débats, et fait oublier les éléments fondamentaux provenant des données de l'expérience de Taylor et les premières idées avancées par la théorie du risque zéro. Il indique également que les découvertes récentes des neurosciences dans le domaine des émotions, et en particulier la théorie des marqueurs somatiques de Damasio [DAM94], ont été ignorées dans ce champ de recherche et qu'il serait intéressant de les rassembler en un modèle plus récent : le modèle du moniteur de risque. L'une des principales critiques de Vaa à l'égard du modèle d'homéostasie du risque [VAA07] concerne sa nature numérique pour laquelle nous n'avons, selon lui, aucune donnée tendant à montrer l'existence de procédures calculatoires dans l'évaluation du risque subjectif en conduite automobile. Il formule ainsi sa critique : Le modèle d'homéostasie du risque ne saisit ni n'imite la dynamique variée de la pensée et des sentiments, les 'courants de la conscience', les fluctuations des états automatisés mélangés aux pensées qui vont et viennent et qui sont si caractéristiques de la conduite au quotidien (p.53). Toutefois, malgré cette critique, Vaa reconnâ̂t au modèle de Wilde, l'intérêt et l'apport de la notion de cible, en précisant que, selon lui, ce n'est pas un niveau de risque que le conducteur cible, mais un niveau de ressenti ou un sentiment (target feeling) [VAA11]. Il pose ainsi l'hypothèse suivante : En plus d'éviter les accidents, les conducteurs recherchent une certaine 'sensation cible'. Ce sentiment n'est pas le même chez tous les conducteurs, chaque conduite a un sentiment cible unique, qui n'est pas nécessairement ressenti consciemment. Les cibles peuvent être définies et caractérisées par une dimension émotionnelle, positive ou négative. ([VAA14], p.118).

Pour justifier son recours aux ressentis dans les processus de décision et d'évaluation subjective du risque en situation de conduite, Vaa s'appuie sur les travaux de Damasio et en particulier sur sa théorie des marqueurs somatiques. Cette théorie stipule que le processus de prise de décision, et en particulier de décision impliquant une évaluation des risques, n'est pas uniquement basé sur un examen rationnel 
où l'émotion et les ressentis n'ont aucun rôle à jouer. Il montre que lors de la prise de décision, les informations du système nerveux autonome en provenance du corps, et particulièrement des viscères, sont prises en compte inconsciemment et qu'elles semblent jouer un rôle déterminant dans l'évitement de solutions inappropriées ou dangereuses. Ainsi, selon Damasio, le corps constituerait une source d'information pouvant marquer positivement ou négativement les solutions envisagées, ce qui contribuerait notamment à réduire considérablement le nombre de solutions à examiner consciemment. En se basant sur ce principe, Vaa propose qu'en situation de conduite, le corps servirait de moniteur de risque, en envoyant des informations de type marqueurs somatiques pour évaluer le risque associé aux éventuelles décisions à prendre. Selon Vaa, le moniteur de risque permettrait au conducteur de se maintenir dans la plage de ressentis cible. Il faut bien noter ici que Vaa, comme Damasio, adoptent des définitions particulières des termes émotion et ressenti. Dans leur approche, les émotions sont restreintes aux réactions neurophysiologiques somatiques, tandis que les ressentis (feelings) sont relatifs aux aspects conscients des émotions. La figure 11 présente l'organisation générale du modèle de Vaa.

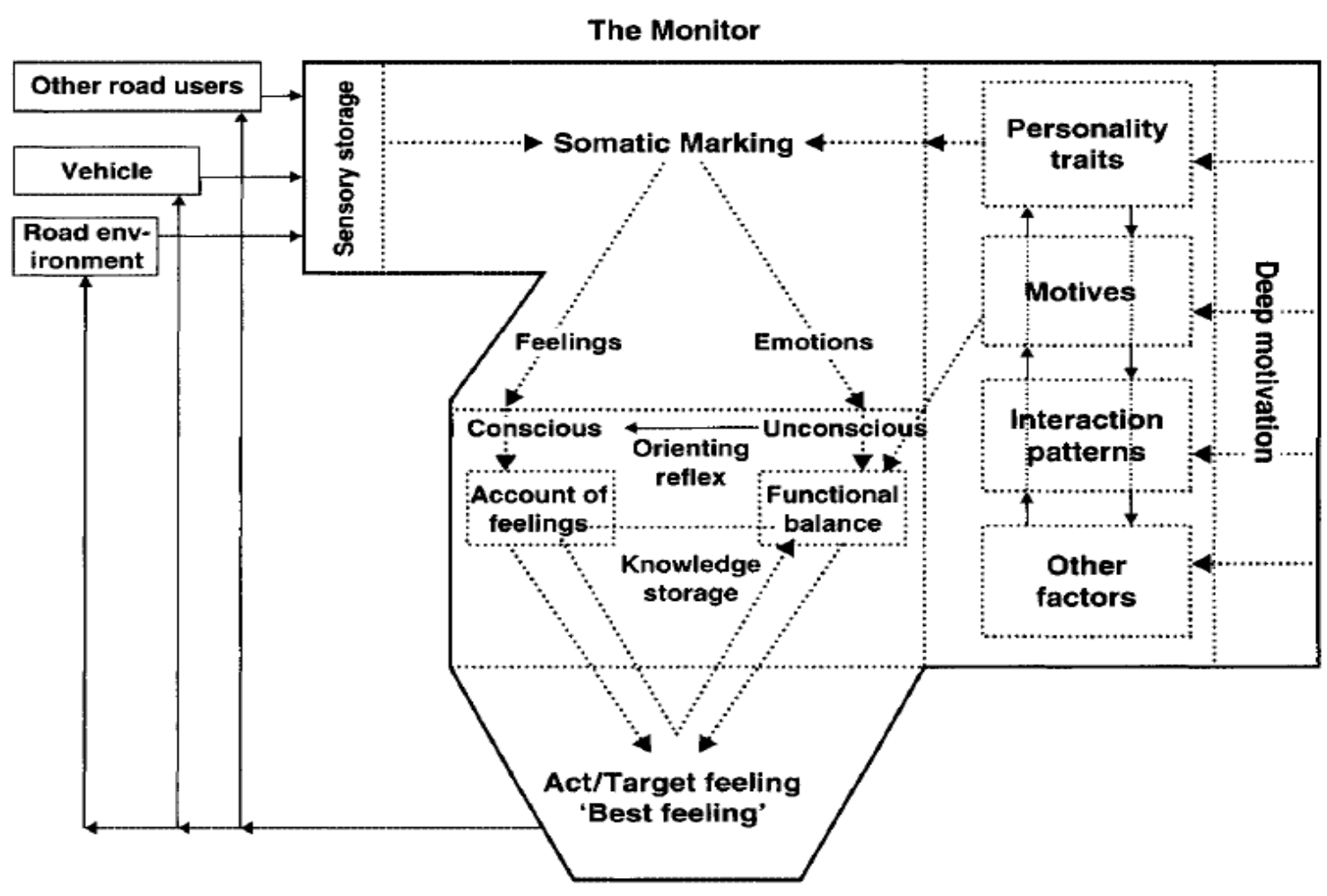

Figure 11. : Modèle du moniteur de risque [VAA07]

Cette distinction entre les aspects conscients et inconscients des processus relatifs à l'évaluation subjective du risque, n'est pas sans évoquer celle faite entre les aspects implicites et explicites et la notion d'émergence dans le cadre du modèle COSMODRIVE dans le cadre duquel a été développée la notion de conscience du risque et qui sera l'objet de la prochaine section.

\section{De la perception du risque à la conscience du risque dans cadre du modèle COSMODRIVE (2010)}

Bellet et Banet [BEL12] à l'issue de leurs travaux sur la question de l'évaluation subjective du risque en situation de conduite chez les motocyclistes, notent un problème terminologique autant que théorique, 
concernant la notion de perception $d u$ risque. Selon ces auteurs, ce que perçoit le conducteur, ce sont des événements, et ce n'est que dans l'analyse de la menace que font peser ces événements sur lui, que le conducteur évalue et se représente le risque. En s'appuyant sur les travaux de Grayson et al. [GRA03], qui distinguent les dangers des risques (les premiers étant la cause des seconds), et en combinaison avec ceux d'Endsley [END95] sur la conscience de la situation, ils proposent la notion de Conscience du risque. Ils définissent cette nouvelle conception comme "une extension de la théorie de la conscience de la situation aux situations de conduite risquées” [BEL12], p.154). Ils justifient cette nouvelle terminologie en indiquant que : "Clairement, la perception n'est pas suffisante pour la conscience du risque : les motocyclistes commelesconducteurs doivent également mentalement évaluer la criticité de la situation en considérant les actions qu'ils sont actuellement en train de mettre en place, et en tenant compte de leurs propres capacités à gérer le risque situationnel” ([BEL12], p.156). Cette conception ainsi que la terminologie qui en émane étant plus précises nous les adopterons désormais.

Dans sa thèse, en 2010, Banet précise cette conception et propose un modèle cadre pour l'analyse de l'évaluation du risque chez le motocycliste. Ce modèle cadre, basé sur le cycle perception - cognition action utilisé pour le développement du modèle COSMODRIVE est représenté schématiquement dans la figure 12 .

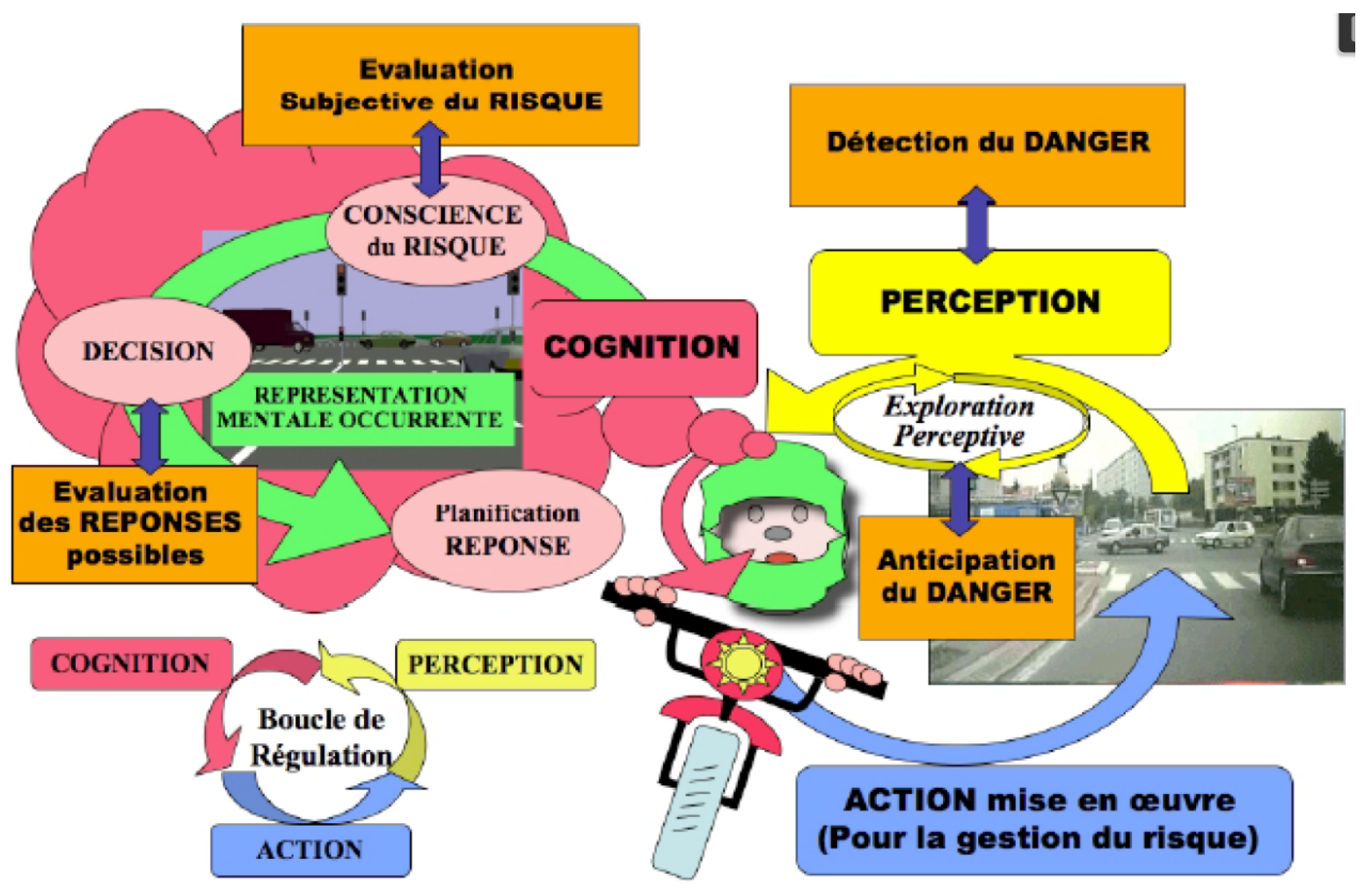

Figure 12. : Modèle cadre pour l'analyse de la conscience du risque [BAN10]

L'idée générale de ce modèle est d'indiquer les différentes étapes et processus nécessaires à l'élaboration de la conscience du risque d'une situation de conduite, en s'appuyant sur les étapes de la boucle perception - cognition - action et les conceptions relatives à l'élaboration de la représentation mentale de la situation de conduite (conscience de la situation). Ainsi, la conscience du risque selon Banet [BAN10] est le fruit d'un "ensemble de processus perceptifs et cognitifs allant de la détection du danger jusqu'à la sélection d'une réponse possible permettant d'éviter l'accident, en passant par l'élaboration d'une représentation mentale occurrente de la situation de conduite et de sa criticité.” (p.133). 
Soulignons également l'intérêt de cette conception cyclique intégrant ces différentes étapes d'évaluation car elle permet non seulement l'utilisation des évaluations subjectives du risque en amont pour guider la prise de décision, mais elle permet également, par la poursuite permanente des processus, d'évaluer la criticité des décisions prises. Cette continuité dans les processus de développement de la conscience du risque ouvre la voie pour le conducteur, d'une part à la gestion du risque et d'autre part à l'identification d'erreurs de prise de décision ou d'implémentation d'actions.

Détaillons à présent ces différentes étapes ainsi que les résultats principaux qui y sont associés.

\subsection{Détection du risque et évaluation subjective du risque}

À chaque étape du cycle, des processus dédiés à l'évaluation subjective du risque peuvent intervenir. Ainsi, au niveau de la phase de perception, les processus d'exploration perceptive incluent la détection (et la recherche) de dangers anticipés. De manière générale, lors de la phase perceptive, la détection du danger est cruciale. En effet, pour une situation impliquant un élément porteur de risque (e.g. véhicule, piéton, objet sur la chaussée, etc.), en cas de non détection de celui-ci, la représentation mentale de la situation sera non seulement erronée mais cette erreur portera précisément sur un élément primordial à considérer dans l'évolution immédiate de la situation.

La distinction de ces deux étapes dissociées de détection du danger et d'évaluation subjective du risque a été mise en évidence par Banet au moyen d'un protocole expérimental mettant en œuvre un outil créé pour comparer différentes populations de motards : CRITIC (Common RIsk awareness measurement meThod for Inter-population Comparisons). L'outil CRITIC est constitué de 25 courtes séquences vidéos présentant des situations de conduites à risque de collision. Pour chaque séquence l'instant à partir duquel le danger est détectable a été marqué par des experts (i.e. instructeurs de moto-école) dans le but de permettre le calcul d'un temps de détection du danger. Les participants de l'étude devaient regarder les séquences et, pour chaque séquence, appuyer sur une touche stoppant la vidéo au moment où la situation devenait critique. La séquence vidéo était ensuite reprise jusqu'à sa fin. Le participant devait alors indiquer le niveau de criticité de la situation sur une échelle continue de 0 à 100 . Les auteurs ont ensuite comparé les résultats fournis par différents types de motards : experts policiers, motards expérimentés, motards sportifs novices et motards débutants. Concernant les temps de détection du danger, deux catégories de groupes de motards se distinguent : les groupes de motards expérimentés et les groupes les moins expérimentés. Les résultats montrent effectivement que l'expérience des motards joue un rôle crucial dans le temps de détection du danger, les motards peu expérimentés étant moins performants que les motards plus aguerris. Concernant l'évaluation subjective du risque situationnel (criticité), les valeurs fournies par les experts policiers étaient considérées comme une référence. La comparaison des valeurs de criticité fournies par les autres groupes de motards laisse apparaitre un groupe se détachant significativement. Si les évaluations de criticité fournies par les motards expérimentés, les motards sportifs novices et les policiers ne se distinguent pas significativement les unes des autres, les motards débutants fournissent quant à eux des évaluations de criticité significativement inférieures.

Ces résultats apportent deux informations cruciales. Ils révèlent, d'un côté, le rôle important de l'expérience de conduite dans l'acquisition des compétences associées à la conscience du risque; de l'autre, ils révèlent que les compétences d'évaluation subjective de la criticité et de détection du danger ne se rapportent pas au même type d'apprentissage. Les résultats obtenus suggèrent donc bien que la détection 
du danger et l'évaluation de la criticité de la situation sont le fruit de processus différents dont les apprentissages sont spécifiques. Ainsi dans cette étude, les motards sportifs novices semblent déjà capables d'évaluer efficacement la criticité générale de la situation sans toutefois être en mesure de détecter le danger aussi rapidement que leurs homologues plus expérimentés. Les motards très débutants, de leur côté, semblent n'avoir ni acquis l'aptitude à détecter rapidement le danger ni acquis celle d'évaluer à son juste niveau la criticité générale de la situation.

Les conclusions de Bellet et Banet [BEL12] semblent donc bien indiquer que détecter le danger ne suffit pas pour obtenir une bonne conscience du risque. Lors de la phase dédiée à la cognition du cycle, la représentation mentale de la situation est élaborée; elle constitue la conscience de la situation. À ce niveau s'élabore une évaluation subjective du risque. Autrement dit, la représentation mentale de la situation se teinte d'une valeur globale de criticité actuelle et anticipée, via l'inclusion des informations perceptives mais également de celles fournies par les connaissances du conducteur. Cette évaluation subjective du risque doit également associer une évaluation des réponses possibles, en tenant compte des capacités perçues du véhicule et de celles disponibles autoévaluées par le conducteur. Ce jugement permet d'alimenter les processus de prise de décision, qui à leur tour, serviront de base à la planification de la réponse. La réponse planifiée sera ensuite mise en action en intervenant sur les commandes du véhicule et inclura une gestion des risques identifiés et évalués. Le plan d'action permet en outre, de produire des anticipations concernant l'évolution de la situation et donc de déterminer des zones de contrôle à explorer perceptivement et pouvant, entre autres, concerner des dangers anticipables. Nous revenons ainsi aux premières étapes du cycle que nous avons décrites.

Dans ce domaine, Banet [BAN10] fournit d'autres résultats venant éclairer l'importance des connaissances de ses propres capacités d'analyse et de gestion dans l'évaluation de la criticité d'une situation de conduite. Pour cela, un autre groupe de motards a été comparé à ceux précédemment cités : les utilisateurs de scooters $125 \mathrm{~cm}^{3}$. Ces motards ont la particularité de ne pas se revendiquer comme appartenant à la catégorie des motards tout en utilisant régulièrement un véhicule similaire. En outre, ils ne bénéficient pas de la formation spécifique que sanctionne l'obtention du permis moto. Lorsqu'ils ont été soumis au protocole CRITIC, ces motards se sont révélés avoir des performances d'évaluation de la criticité des situations de conduite significativement inférieures aux motards experts et expérimentés. Leurs performances dans ce domaine se sont mêmes révélées plus proches de celles des débutants que des jeunes motards sportifs. Ce type de résultats peut être interprété comme le fruit d'une potentielle meilleure connaissance acquise par les motards sportifs au cours de leur formation et par leur recherche de performances durant leurs premiers mois de pratique de la moto sur route.

L'évaluation de la criticité générale de la situation de conduite, repose également sur un certain nombre d'anticipations à propos de l'évolution de la situation de conduite. Ces anticipations se font par le biais du processus de déploiement cognitif au cours duquel les zones enveloppes jouent un rôle particulier dans l'évaluation et la gestion des risques.

\subsection{De la conscience à la gestion du risque : les Zones Enveloppes et les matrices de conflit}

Les Zones Enveloppes (ZE) (concept proposé par Bellet [BEL98], [BEL07]) assurent plusieurs fonctions dans la gestion de la situation de conduite. Elles se rapportent entre autre à la gestion des distances de sécurité avec les autres objets de l'environnement. À ce titre, elles peuvent donc être utilisées lors des 
processus d'évaluation subjective de la criticité situationnelle en s'appuyant sur un processus de déploiement cognitif [BEL09]. En effet, le conducteur projette mentalement les ZE pour lui-même et pour les autres usagers. Cette simulation mentale lui permet d'anticiper les chevauchements de trajectoires et donc les conflits potentiels à venir. Il procède ainsi à un examen des conflits de zones enveloppes prévisibles suivant les différentes évolutions possibles de la situation de conduite. Ce processus de déploiement et d'anticipation va permettre au conducteur d'estimer la criticité de la situation dans son devenir et de prendre des décisions sur les actions les plus appropriées au maintien des conditions de sécurité.

Jean-Charles Bornard [BOR12] propose, en suivant ce raisonnement, une matrice de conflit adaptée aux situations de tourne-à-gauche. La figure 13 illustre cette matrice. Le code couleur utilisé traduit le niveau de risque associé à chaque conflit.

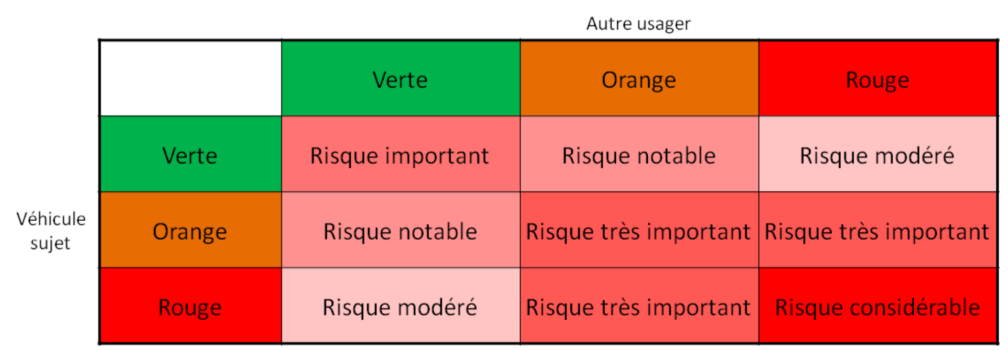

Figure 13. : Exemple de matrice de conflit de Zones Enveloppes en situation de tourne à gauche ([BOR12] p.178)

Sur la base de cette matrice, il est possible d'associer un niveau de risque de collision en fonction de chaque recouvrement entre une Zone Enveloppe du véhicule du conducteur et une Zone Enveloppe du véhicule d'un autre usager. Pour mieux comprendre et interpréter cette matrice de conflit, étudions son application à la manœuvre de tourne-à-gauche telle qu'elle a été étudiée par Jean-Charles Bornard [BOR12].

\subsection{Prise de décision et gestion du risque en situation de tourne à gauche}

Pour étudier l'évaluation du risque et les prises de décisions lors des manœuvres de tourne-à-gauche, Bornard a eu recours à une méthodologie innovante dans laquelle il a utilisé son implémentation du modèle COSMODRIVE pour effectuer des prédictions de conflits de ZE et en déduire des hypothèses opérationnelles de décisions de franchissement de l'intersection. Une fois ces simulations effectuées, il les a confrontées aux décisions et comportements effectifs de conducteurs humains dans la même situation de conduite simulée. Le fait que les simulations du modèle COSMODRIVE et les situations de conduite expérimentales aient eu lieu dans le même environnement simulé garantit une comparabilité des résultats fournis par le modèle et des résultats fournis par les conducteurs humains. Pour effectuer les simulations avec COSMODRIVE Bornard se base sur le schéma de conduite de la manœuvre de tourne-à-gauche, sur une implémentation des ZE et sur une prise en compte de différentes trajectoires et différentes vitesses possibles lors de la manœuvre de franchissement de l'intersection [BEL03].

La figure 14 présente une situation de tourne à gauche telle qu'étudiée par Bornard. Lors de cette manœuvre le conducteur doit s'insérer entre deux véhicules circulant dans le sens opposé (i.e. véhicules A et B); les conflits de ZE ont lieu entre le véhicule du conducteur (i.e. Ego) et le véhicule du trafic arrivant dans la voie en sens opposée (i.e. voiture B). Pour prendre sa décision, il doit estimer la taille 


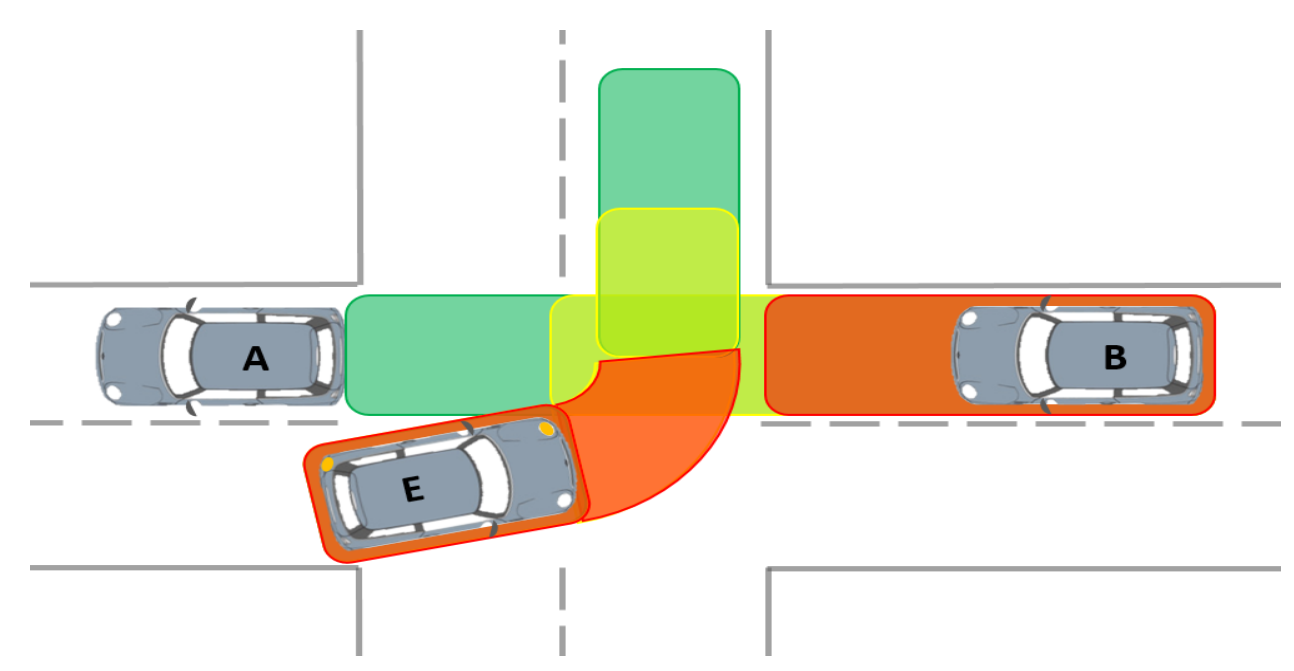

Figure 14. : Illustration d'une situation de tourne à gauche avec conflit de ZE

de l'espace entre deux véhicules dans le trafic arrivant en sens opposé et projeter les zones enveloppes sur le véhicule fermant l'espace, afin d'estimer les conflits potentiels avec ses propres ZE. En fonction du Temps Inter-Véhiculaire (TIV) disponible et de la vitesse à laquelle il réalise la manœuvre de tourne à gauche, les conflits de ZE seront différents. La figure 15 présente les différents types de conflits de ZE possibles suivant le TIV et le temps de franchissement de l'intersection par le conducteur.

\begin{tabular}{|l|l|l|l|}
\hline TIV & $\begin{array}{l}\text { conflit de ZE avec } \\
\mathrm{T}_{\text {min }}\end{array}$ & $\begin{array}{l}\text { conflit de ZE avec } \\
\mathrm{T} \text { Moy }\end{array}$ & $\begin{array}{l}\text { conflit de ZE avec } \\
\mathrm{T} \text { MAx }\end{array}$ \\
\hline 0,6 & Accident & Accident & Accident \\
1,2 & Rouge-Rouge & Accident & Accident \\
1,8 & Rouge-Orange & Rouge-Rouge & Accident \\
2,3 & Rouge-Verte & Rouge-Orange & Accident \\
2,6 & Rouge-Aucun & Rouge-Verte & Rouge-Rouge \\
3,0 & Rouge-Aucun & Rouge-Verte & Rouge-Orange \\
3,3 & Aucun & Aucun & Rouge-Orange \\
3,6 & Aucun & Aucun & Rouge-Verte \\
4,0 & Aucun & Aucun & Rouge-Verte \\
5,0 & Aucun & Aucun & Aucun \\
6,0 & Aucun & Aucun & Aucun \\
\hline
\end{tabular}

Figure 15. : Conflits de Zones Enveloppes en situation de Tourne-à-gauche (issu de [BOR2012], p.178)

La matrice de conflit permet d'interpréter les conflits présentés dans la figure 15. Ainsi, un conflit rouge-vert représentera un risque modéré, un conflit rouge-orange un risque très important et un conflit rouge-rouge un risque considérable, les autres cas aboutissant soit à un accident, soit à une absence de conflit. En confrontant ses hypothèses aux décisions et comportements des conducteurs, Bornard montre qu'il existe non seulement un lien entre la taille de l'espace dans le trafic et le pourcentage de décision de franchissement des conducteurs, mais également que ce lien est bien expliqué par la matrice de conflits de Zones Enveloppes. La figure 16 montre les conflits de ZE associés aux pourcentages de franchissement de l'intersection tels qu'ils ont été enregistrés auprès des participants lors de son expérimentation.

De plus, lors de son étude, Jean-Charles Bornard demandait aux participants d'évaluer la criticité des situations de tourne-à-gauche. Il obtient alors pour chaque taille de gap dans le trafic, une estimation sur une échelle continue de 0 à 100 , du risque subjectivement évalué par les participants. Les résultats montrent alors une forte corrélation entre la taille de l'espace et la criticité subjectivement estimée par les participants. Le graphique de la figure 17 traduit cette relation. 


\begin{tabular}{|l|l|l|l|}
\hline TIV & $\begin{array}{l}\text { conflit de ZE avec } \\
\mathrm{T}_{\text {min }}\end{array}$ & $\begin{array}{l}\text { conflit de ZE avec } \\
\mathrm{T}_{\text {Moy }}\end{array}$ & $\begin{array}{l}\text { conflit de ZE avec } \\
\mathrm{T}_{\text {MAX }}\end{array}$ \\
\hline 0,6 & Accident & Accident & Accident \\
1,2 & Rouge-Rouge & Accident & Accident \\
1,8 & Rouge-Orange & Rouge-Rouge & Accident \\
2,3 & Rouge-Verte & Rouge-Orange & Accident \\
2,6 & Rouge-Aucun & Rouge-Verte & Rouge-Rouge \\
3,0 & Rouge-Aucun & Rouge-Verte & Rouge-Orange \\
3,3 & Aucun & Aucun & Rouge-Orange \\
3,6 & Aucun & Aucun & Rouge-Verte \\
4,0 & Aucun & Aucun & Rouge-Verte \\
5,0 & Aucun & Aucun & Aucun \\
6,0 & Aucun & Aucun & Aucun \\
\hline
\end{tabular}

Figure 16. : Conflits de Zones Enveloppes et pourcentages de décisions de franchissement (issu de [BOR2012], p.178)

\begin{tabular}{|l|l|}
\hline TIV & $\begin{array}{l}\text { moyenne } \\
\text { criticité }\end{array}$ \\
\hline 0,6 & 96,13 \\
1,2 & 87,58 \\
1,8 & 83,84 \\
2,3 & 74,02 \\
2,6 & 64,89 \\
3 & 60,98 \\
3,3 & 48,29 \\
3,6 & 43,49 \\
4 & 36,24 \\
5 & 20 \\
6 & 10,63 \\
\hline
\end{tabular}

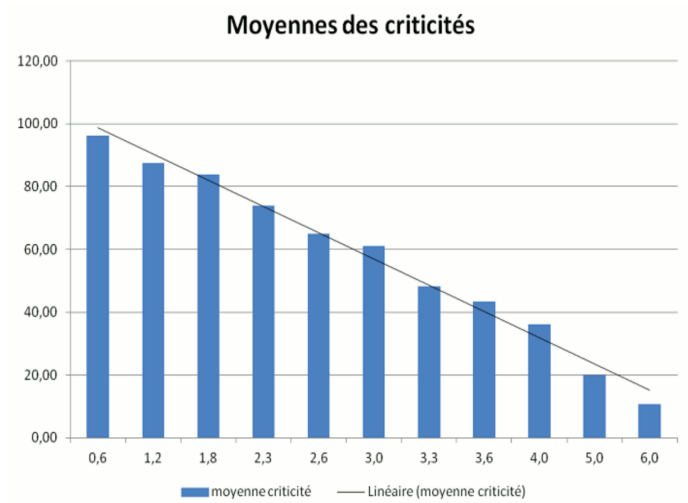

Figure 17. : Criticité moyenne du tourne-à-gauche suivant la taille des gaps dans le trafic (issu de [BOR2012], p.181)

L'auteur montre que ce digramme en basse présente plusieurs « sauts » au niveau de certaines valeurs d'espaces dans le trafic correspondant aux changements de types de conflits dans la matrice de conflits. Cet élément est donc en faveur du caractère explicatif de la matrice de conflits autant en ce qui concerne les décisions des conducteurs qu'en ce qui se rapporte aux valeurs de criticité de la situation de tourneà-gauche.

Nous disposons donc par le biais de ces résultats, une meilleure compréhension de l'utilité des Zones Enveloppe pour permettre au conducteur de produire une bonne conscience du risque situationnel. Toutefois, nous ne savons pas si cette conception permet d'anticiper d'autres types de décisions de la même manière en particulier dans le contexte d'autres types de manœuvres. Qu'en est-il par exemple des situations de changement de voie?

\section{Conclusion}

Tout au long de cet article récapitulatif, nous avons brossé un portrait global des travaux concernant l'évaluation subjective du risque plus particulièrement dans le domaine de la cognition du conducteur. Après avoir effectué un court bilan de la notion même de risque et de la manière dont elle a été développée et étudiée dans l'histoire des sciences, nous nous sommes concentrés sur les travaux dédiés à l'étude de l'évaluation subjective du risque (perception du risque) de manière générale par les individus. Ces travaux nous ont ensuite servi de base pour aborder la question de l'évaluation subjective du risque par 
les conducteurs en situation de conduite. Nous avons ainsi pu constater qu'il existe à la fois des liens entre les théories générales de la perception du risque et celles dédiées à l'évaluation du risque par le conducteur, mais que ces théories générales ne permettent pas de rendre compte en détail, des processus cognitifs mis en œuvre par les conducteurs en situation. Nous avons également vu que les modèles développés au cours des dernières décennies dans le cadre de l'étude de la cognition du conducteur, ont progressivement cherché à intégrer l'évaluation du risque situationnel aux modèles cognitifs de l'activité de conduite pour aboutir à la notion de conscience du risque. De nombreuses questions restent toutefois en suspens concernant à la fois les notions de risque cible, d'évaluation situationnelle ou d'applicabilité des Zones Enveloppes à une grande variété de situations. Par ailleurs, certaines questions demeurent également concernant les liens entre les risques accidentologiques objectifs et l'évaluation subjective qui en est faite par les conducteurs. En effet, les liens entre la dimension statistique accidentologique et l'évaluation subjective faite par les conducteurs n'est pas encore parfaitement élucidée, et nous pourrions envisager d'interroger l'hypothèse du cerveau statisticien pour chercher à éclairer cette problématique. Cette hypothèse [DEH11], [TEN11], proposant que le cerveau procède hiérarchiquement à des inférences statistiques permettant un apprentissage et une généralisation rapide pourrait possiblement rendre compte des questions d'apprentissage sans accidents et à partir d'événements rares. Elle pourrait également être compatible avec les conceptions récentes de Zones Enveloppes et de schéma de conduite mais aussi de rendre compte de la dimension heuristique des évaluations qui sont effectuées en direct par les conducteurs lors de la conduite. Enfin, une meilleure intégration des mécanismes émotionnels et de leur rôle dans l'évaluation subjective du risque devrait être envisagée dans la mesure où certains travaux que nous avons évoqués, montrent que les émotions et les affects semblent jouer un rôle important dans l'évaluation du risque et les décisions prises par les conducteurs. De nombreuses recherches seront donc encore nécessaires pour établir une modélisation pertinente à l'évaluation subjective du risque chez les conducteurs.

\section{Bibliographie}

[ADA70] AdAms J. R, «Personality Variables Associated with Traffic Accidents. » Behavioral Research in Highway Safety $1, \mathrm{n}^{\circ} 1$ (1970).

[AMA96] Amalberti R., La Conduite Des Systèmes à Risques. PUF Paris, 1996.

[ASC87] Aschenbrenner K. M., Biehl B., Wurm G. W., «Einfluss Der Risikokompensation Auf Die Wirkung von Verkehrssicherheitsmassnahmen Am Beispiel Abs. » Bundesanstalt für Straßenwesen (BASt), 1987.

[BAN10] Banet A., Conscience Du Risque et Attitudes Face Aux Risques Chez Les Motocyclistes. PhD thesis. 2010. [BEL98] Bellet T., Modélisation et Simulation Cognitive de L'opérateur Humain : Une Application à La Conduite Automobile. PhD thesis. 1998.

[Bel03] Bellet T., Tattegrain-Veste H., Chapon A., Bruyas M. P., Pachiaudi G., Deuleurance P., GuilHON V., «L'ingénierie Cognitive : IHM et Cognition. » Paris : Hermes Sciences., 2003.

[Bel07] Bellet T., Bailly-Asuni B., Mayenobe P., Georgeon O., « Cognitive modelling and computational simulation of drivers mental activities. » Critical Issues in Advanced Automotive Systems and Human-Centred Design, pp.317-345., (2007).

[Bel09] Bellet T., Bailly-Asuni B., Mayenobe P., Banet A., «A Theoretical and Methodological Framework for Studying and Modelling Drivers' Mental Representations. » Safety Science 47, n 9 (2009).

[BER59] Bernard C., Leçons Sur Les Propriétés Physiologiques et Les Altérations Pathologiques Des Liquides de L'organisme. Paris : J.-B. Baillière 1859.

[BER38] Bernoulli D., Specimen Theoriae Novae de Mensura Sortis. 1738.

[BOR12] Bornard J-C., Développement d'un Modèle Du Conducteur Automobile : De La Modélisation Cognitive à La Simulation Numérique. PhD thesis. 2012. 
[CAD05] Cadet B., Kouabénan D. R., «Évaluer et Modéliser Les Risques : Apports et Limites de Différents Paradigmes Dans Le Diagnostic de Sécurité. »Le Travail Humain 68, ${ }^{\circ} 1$ (2005).

[CAN29] Cannon W. B., «Organization for Physiological Homeostasis. » Physiological Reviews 9, n 3 (1929).

[COW66] Cownie A. R., CALDERwood J. H., «Feedback in Accident Control. » Journal of the Operational Research Society $17, \mathrm{n}^{\circ} 3$ (1966).

[DAM94] Damasio A. R., L'erreur de Descartes Paris : Odile Jacob. 1994.

[END95] EndSLEY M. R., «Measurement of Situation Awareness in Dynamic Systems. » Human Factors : The Journal of the Human Factors and Ergonomics Society 37, $\mathrm{n}^{\circ} 1$ (1995).

[FUL84] FULLER R., «A Conceptualization of Driving Behaviour as Threat Avoidance.» Ergonomics 27, $\mathrm{n}^{\circ} 11$ (1984).

[FUL00] FulLER R., «The Task-Capability Interface Model of the Driving Process. » Recherche - Transport - Sécurité 66, (2000).

[FUL05] FUlleR R., «Towards a General Theory of Driver Behaviour. » Accident Analysis \& Prevention 37, $\mathrm{n}^{\circ} 3$ (2005).

[FUl08] Fuller R., McHugh C., Pender S., «Task Difficulty and Risk in the Determination of Driver Behaviour. » Revue Européenne de Psychologie Appliquée / European Review of Applied Psychology 58, $\mathrm{n}^{\circ} 1$ (2008).

[FUL11] Fuller R., Driver Control Theory. In Handbook of Traffic Psychology, 13-26. Elsevier. 2011.

[GRa03] Grayson G. B., Maycock G., Groeger J. A., Hammond S. M., Field D. T., Risk, Hazard Perception and Perceived Control. 2003.

[HeR08] Hermand D., Chauvin B. «Contribution Du Paradigme Psychométrique à L'étude de La Perception Des Risques : Une Revue de Littérature de 1978 à 2005. » L'année Psychologique 108, n 2 (2008).

[HOG80] Hogarth R. M., Judgment and Choice. New York : Wiley. 1980.

[KAH11] Kahneman D., Système 1 Système 2 : Les Deux Vitesses de La Pensée. Flammarion. 2011.

[LEW09] Lewis-Evans B., Rothengatter T. «Task Difficulty, Risk, Effort and Comfort in a Simulated Driving Task-Implications for Risk Allostasis Theory. » Accident Analysis \& Prevention 41, $\mathrm{n}^{\circ} 5$ (2009).

[MAC05] Macgill S. M., SiU Y. L., «A New Paradigm for Risk Analysis. » Futures 37, n 10 (2005).

[MCK82] McKenna F. P., «The Human Factor in Driving Accidents an Overview of Approaches and Problems. » Ergonomics 25, $\mathrm{n}^{\circ} 10$ (1982).

[MIC85] Michon J. A., Human Behavior and Traffic Safety. In Human Behavior and Traffic Safety. New York : New York Plenum Press. 1985.

[NAA74] NäÄTÄNEn R., Summala H., «Model for the Role of Motivational Factors in Drivers' Decision-Making. » Accident Analysis \& Prevention 6 (1974).

[NAA76] NäÄTÄnen R, Summala H., Road-User Behaviour and Traffic Accidents. North-Holland Publishing Company. 1976.

[PLA71] PlaneK T. W., «Driver Education Research in the United-States-Can the New Directions Be Supported?» In Proceedings of the International Symposium on Psychological Aspects of Driver Behaviour. 1971.

[RAU01] Raufaste E., Hilton D. J., «Les Mécanismes de La Décision Face Au Risque. » Alliage 48 (2001).

[REA90] REASON J., Human Error. UK : Cambridge University Press. 1990.

[SLO87] SlOVIC P., « Perception of Risk. » Science 236, n 4799 (1987).

[SlO04] Slovic P., Finucane M. L., Peters E., MacGregor D. G., «Risk as Analysis and Risk as Feelings : Some Thoughts About Affect, Reason, Risk, and Rationality. »Risk Analysis 24, n 2 (2004).

[SlO07] Slovic P., Finucane M. L., Peters E., MacGregor D. G., «The Affect Heuristic. » European Journal of Operational Research 177, $\mathrm{n}^{\circ} 3$ (2007).

[STA69] STARR C., «Social Benefit Versus Technological Risk. » Science 165, n 3899 (1969).

[SUM88] Summala H., «Risk Control Is Not Risk Adjustment : The Zero-Risk Theory of Driver Behaviour and Its Implications. » Ergonomics 31, n 4 (1988).

[TAY64] TAYLOR D. H., « Drivers' Galvanic Skin Response and the Risk of Accident. » Ergonomics 7, n 4 (1964).

[TEN11] Tenenbaum J. B., Kemp C., Griffiths T. L., Goodman N. D., « How to Grow a Mind : Statistics, Structure, and Abstraction. » Science 331, $\mathrm{n}^{\circ} 6022$ (2011).

[VAA01] VAA T., «Cognition and Emotion in Driver Behaviour Models : Some Critical Viewpoints. » In Proceedings of the 14th Ictct Workshop. 2001. 
[VAA14] VAA T. «From Gibson and Crooks to Damasio : The Role of Psychology in the Development of Driver Behaviour Models. » Transportation Research Part F : Traffic Psychology and Behaviour 25, (2014).

[VAA07] VAA T., Modelling Driver Behaviour on Basis of Emotions and Feelings : Intelligent Transport Systems and Behavioural Adaptations. In Modelling Driver Behaviour in Automotive Environments : Critical Issues in Driver Interactions with Intelligent Transport Systems, London : Springer. 2007.

[VAA11] VAA T., Drivers' Information Processing, Decision-Making and the Role of Emotions : Predictions of the Risk Monitor Model. In Human Modelling in Assisted Transportation, Milano : Springer. 2011.

[VAN88] Van Der Molen H. H., Bötticher A. M. T., «A Hierarchical Risk Model for Traffic Participants. » Ergonomics 31, $\mathrm{n}^{\circ} 4$ (1988).

[WIL82] WILDE G. J. S., «The Theory of Risk Homeostasis : Implications for Safety and Health. » Risk Analysis 2, $\mathrm{n}^{\circ}$ 4 (1982).

[WIL14] Wilde G. J. S., Target Risk 3 : Risk Homeostasis in Everyday Life. Digital Edition. http ://riskhomeostasis.org/links-to-target-risk. 2014. 